PREPARED FOR THE U.S. DEPARTMENT OF ENERGY, UNDER CONTRACT DE-AC02-76CH03073

PPPL-3599

PPPL-3599

UC-70

Simulations of Electron Transport in Laser Hot Spots

by

S. Brunner and E. Valeo

August 2001

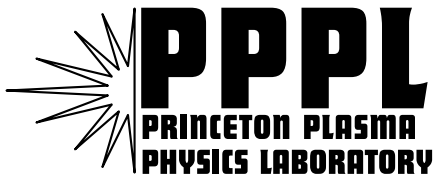

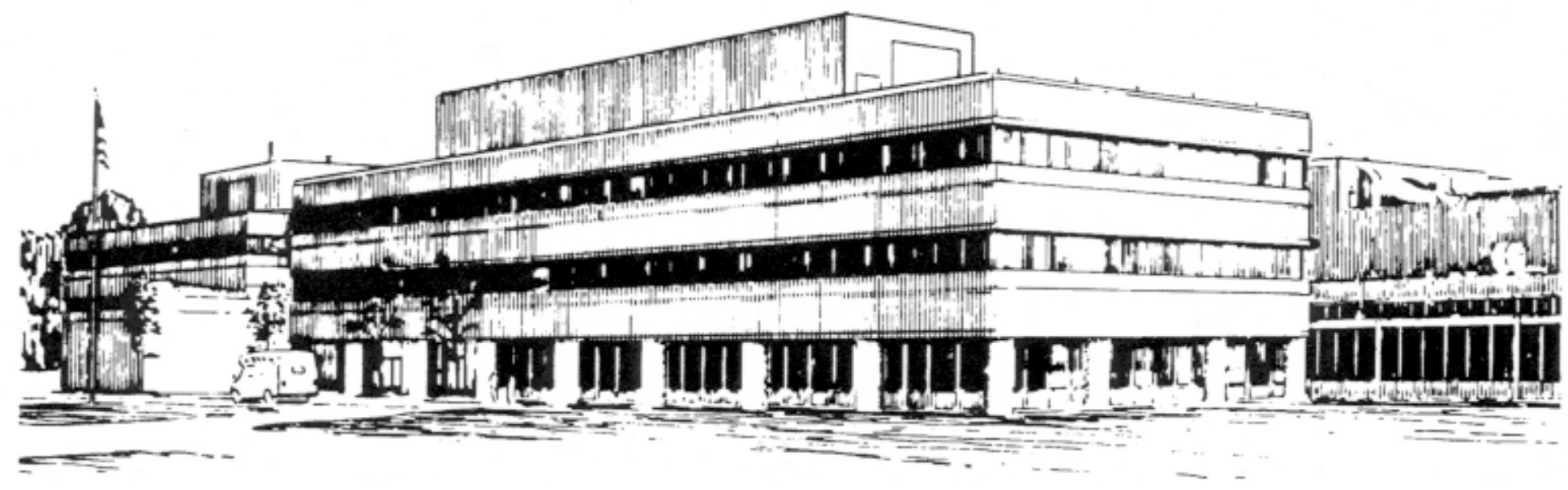

PRINCETON PLASMA PHYSICS LABORATORY PRINCETON UNIVERSITY, PRINCETON, NEW JERSEY 


\section{PPPL Reports Disclaimer}

This report was prepared as an account of work sponsored by an agency of the United States Government. Neither the United States Government nor any agency thereof, nor any of their employees, makes any warranty, express or implied, or assumes any legal liability or responsibility for the accuracy, completeness, or usefulness of any information, apparatus, product, or process disclosed, or represents that its use would not infringe privately owned rights. Reference herein to any specific commercial product, process, or service by trade name, trademark, manufacturer, or otherwise, does not necessarily constitute or imply its endorsement, recommendation, or favoring by the United States Government or any agency thereof. The views and opinions of authors expressed herein do not necessarily state or reflect those of the United States Government or any agency thereof.

\section{Availability}

This report is posted on the U.S. Department of Energy's Princeton Plasma Physics Laboratory Publications and Reports web site in Calendar Year 2001. The home page for PPPL Reports and Publications is: http://www.pppl.gov/pub_report/

DOE and DOE Contractors can obtain copies of this report from:

U.S. Department of Energy

Office of Scientific and Technical Information

DOE Technical Information Services (DTIS)

P.O. Box 62

Oak Ridge, TN 37831

Telephone: (865) 576-8401

Fax: (865) 576-5728

Email: reports@adonis.osti.gov

This report is available to the general public from:

National Technical Information Service

U.S. Department of Commerce

5285 Port Royal Road

Springfield, VA 22161

Telephone: 1-800-553-6847 or

(703) 605-6000

Fax: (703) 321-8547

Internet: http://www.ntis.gov/ordering.htm 


\title{
Simulations of Electron Transport in Laser Hot Spots
}

\author{
S. Brunner* and E. Valeo \\ Princeton Plasma Physics Laboratory, P.O. Box 451, Princeton, New Jersey 08543-0451
}

\begin{abstract}
Simulations of electron transport are carried out by solving the Fokker-Planck equation in the diffusive approximation. The system of a single laser hot spot, with open boundary conditions, is systematically studied by performing a scan over a wide range of the two relevant parameters: (1) Ratio of the stopping length over the width of the hot spot. (2) Relative importance of the heating through inverse Bremsstrahlung compared to the thermalization through self-collisions. As for uniform illumination [J. P. Matte et al., Plasma Phys. Controlled Fusion 30, 1665 (1988)], the bulk of the velocity distribution functions (VDFs) present a super-Gaussian dependence. However, as a result of spatial transport, the tails are observed to be well represented by a Maxwellian. A similar dependence of the distributions is also found for multiple hot spot systems. For its relevance with respect to stimulated Raman scattering, the linear Landau damping of the electron plasma wave is estimated for such VDFs. Finally, the non-linear Fokker-Planck simulations of the single laser hot spot system are also compared to the results obtained with the linear non-local hydrodynamic approach [A. V. Brantov et al., Phys. Plasmas 5, 2742 (1998)], thus providing a quantitative limit to the latter method: The hydrodynamic approach presents more than $10 \%$ inaccuracy in the presence of temperature variations of the order $\Delta T / T \gtrsim 1 \%$, and similar
\end{abstract}

\footnotetext{
*E-mail: sbrunner@pppl.gov
} 
levels of deformation of the Gaussian shape of the Maxwellian background. 52.65.Ff, 52.38.Dx

Typeset using REVTEX 


\section{INTRODUCTION}

The non-Maxwellian electron velocity distributions, resulting from the non-classical drive and transport under laser-fusion relevant conditions, may significantly affect the dispersion and damping of the different waves present in the plasma. This in turn may alter the thresholds and gains of parametric instabilities, such as stimulated Raman scattering (SRS) and stimulated Brillouin scattering (SBS), involving the plasma wave and the ion acoustic wave respectively. This issue has been addressed in the past by Bychenkov et al. ${ }^{1}$ as well as Afeyan et al..2

The systematic characterization of the non-Maxwellian electron velocity distribution functions was performed by Matte et $a .^{3}{ }^{3}$ in the case of uniform laser illumination, i.e. neglecting spatial transport. In this study, the competition between inverse Bremsstrahlung, which pushes the distribution towards a super-Gaussian of the form $f \sim \exp \left[-(v / u)^{5}\right],{ }^{4}$ and the thermalization through self-collisions was considered. It was shown, that the distribution is well represented, at all energies, by a so-called Dum-Langdon-Matte (DLM) type function $f_{\mathrm{DLM}} \sim \exp \left[-(v / u)^{n}\right]$, where the parameter $n$ takes intermediate values between $n=2$ (a Maxwellian), in the absence of drive, and $n=5$, when inverse Bremsstrahlung dominates. As a practical result, a relation $n=n(\alpha)$ was established between the DLM exponent $n$ and the relevant parameter $\alpha=Z\left(v_{\mathrm{os}} / v_{\mathrm{th}}\right)^{2}$, measuring the relative importance of inverse Bremsstrahlung compared to self-collisions. Here, Z is the degree of ionization, $v_{\text {os }}$ the quiver velocity, and $v_{\text {th }}$ the thermal velocity.

A question, which naturally arises in this context, concerns the validity of Matte's local relation in the case of an inhomogeneous plasma, where spatial transport takes place. Inhomogeneities are expected to particularly affect the tails of the distribution, formed by the highly mobile particles. In reference 3 , the relation $n=n(\alpha)$ was in fact already tested in a simulation of a non-uniform plasma, modeling the laser absorption in an underdense region, and the subsequent heat transport into the overdense region. As expected, the local relation failed to correctly represent the distributions near the critical surface, where the plasma 
parameters vary rapidly and spatial transport is therefore important. The representation of the electron distributions with DLM functions was also applied in reference 2, in the particular case of a plasma heated by a realistic intensity profile of a random phase plate (RPP) laser beam.

To our knowledge, besides these few particular cases, no systematic study of the effect of spatial transport on the electron velocity distributions has been published. We therefore propose here to carry out a somewhat more detailed characterization of the distributions under conditions of spatial transport. Due to its relative simplicity, the case of a single laser hot spot seems well suited for this purpose. Indeed, besides $\alpha=Z\left(v_{\mathrm{os}} / v_{\mathrm{th}}\right)^{2}$, measuring the level of drive, such a system is characterized by essentially one additional parameter, measuring the level of spatial transport. This parameter can be chosen as the ratio $\lambda / \Delta r$ between the effective mean free path $\lambda$, and the radial focal width $\Delta r$. By carrying out a scan over this two-dimensional parameter space, one can thus obtain a complete picture of the dynamics of a single hot spot in all possible regimes. The ongoing single laser hot spot experiment at the Los Alamos National Laboratory (LANL) ${ }^{5}$ provides further incentive for studying such a system.

The case of a single hot spot is also ideal for testing the limits of the non-local hydrodynamic approach. ${ }^{6-8}$ This method provides closure relations to the fluid equations for all regimes of collisionality, but is only valid in the linear regime of small perturbations with respect to a given background plasma, assumed to be Maxwellian. Defining quantitatively the limits of the linear regime remains an open issue, and we shall attempt to address this point in this context by using our non-linear Fokker-Planck simulations as a reference.

The single hot spot naturally remains a particular case of an inhomogeneous system. In fact, the relevance of such results even to the case of a multiple hot spot system is, a priori, not evident. For this reason, we shall also present simulations of a plasma heated by multiple heat sources, and show to what extent the single source results may still be applied.

The rest of the paper is organized as follows. The equations solved in the simulations are derived in Sec. II, and their numerical implementation is briefly described in Sec. III. 
The parameter scan for the single laser hot spot system is then presented in Sec. IV. The actual parameter range considered is defined in Sec. IV A, the evolution of temperature is discussed in Sec. IV B, the appropriate fitting of the velocity distributions is addressed in Sec. IV C. To estimate the effect of the non-Maxwellian distributions on Landau damping, these fits are then used in Sec. IV D for solving the dispersion relation of plasma waves. Section V presents the comparisons between the Fokker-Planck calculations and the nonlocal hydrodynamic results. Simulations of a multiple hot spot system are discussed in Sec. VI. We finally conclude in Sec. VII.

\section{PHYSICAL MODEL}

The transport of electrons is described by the corresponding Fokker-Planck equation:

$$
\frac{\partial f}{\partial t}+\boldsymbol{v} \cdot \frac{\partial f}{\partial \boldsymbol{x}}+\frac{(-e)}{m} \boldsymbol{E} \cdot \frac{\partial f}{\partial \boldsymbol{v}}=-\left\{C_{e e}[f, f]+C_{e i} f\right\}
$$

where $\boldsymbol{E}$ stands for the self-consistent electrostatic field ensuring quasineutrality, $C_{e e}$ for the Landau electron-electron self-collision operator, and $C_{e i}$ for the Lorentz electron-ion collision operator. For the simulations presented in this paper, ions have been kept fixed. To lighten notations, physical quantities relative to electrons will often not be explicitly labeled as such, unless required for clarity.

For solving the Fokker-Planck equation, a high ionic charge $Z$ is assumed, such that the electron-ion mean free path $\lambda_{\text {ei }}$ is small compared to any characteristic scale-length $L$ of the system. In this ordering, the electron stopping length ${ }^{9,10} \lambda_{\epsilon} \sim\left(\bar{\lambda}_{e e} \bar{\lambda}_{e i}\right)^{1 / 2} \sim Z^{1 / 2} \bar{\lambda}_{e i}$, can however be such that $\lambda_{\epsilon} / L \sim 1$, thus potentially giving rise to nonlocal transport. Equation (1) can then be solved in an expansion series with respect to the small parameter $\epsilon=\lambda_{\text {ei }} / L$ :

$$
f(\boldsymbol{x}, \boldsymbol{v} ; t)=\sum_{n=0} f_{n}(\boldsymbol{x}, \boldsymbol{v} ; t), \quad \text { with } \quad f_{n} \sim \epsilon^{n}
$$

To lowest order, the electron dynamics are dominated by the pitch-angle scattering off of ions: 


$$
0=-C_{e i} f_{0}
$$

As a result, $f_{0}=f_{0}(\boldsymbol{x}, v ; t)$ is an isotropic function of velocity. Expansion (2) is only considered up to the next order term $f_{1}$, which represents the lowest order anisotropy in velocity, and arises from the convection both in space and velocity. The corresponding equation is given by

$$
\frac{\partial f_{1}}{\partial t}+\boldsymbol{v} \cdot \frac{\partial f_{0}}{\partial \boldsymbol{x}}+\frac{(-e)}{m} \boldsymbol{E} \cdot \frac{\boldsymbol{v}}{v} \frac{\partial f_{0}}{\partial v}=-\left\{C_{e e}\left[f_{0}, f_{1}\right]+C_{e e}\left[f_{1}, f_{0}\right]+C_{e i} f_{1}\right\}
$$

Invoking again high $Z, \partial f_{1} / \partial t$ and the $C_{e e}$ terms appearing in (3) are small compared to $C_{e i} f_{1}$, and can therefore be neglected. It is then straightforward to solve for $f_{1}$ :

$$
f_{1}=-\frac{\boldsymbol{v}}{2 \nu_{e i}(v)} \cdot\left[\frac{\partial f_{0}}{\partial \boldsymbol{x}}+\frac{(-e)}{m} \boldsymbol{E} \frac{1}{v} \frac{\partial f_{0}}{\partial v}\right],
$$

where $\nu_{\mathrm{ei}}(v)=e^{2} N_{i}(Z e)^{2} \ln \Lambda / 8 \pi \epsilon_{0}^{2} m^{2} v^{3}$ is the electron-ion collision frequency.

After having inserted in Eq.(1) the relation $f \simeq f_{0}+f_{1}$, with $f_{1}$ given by Eq.(4), and averaged over the solid angle in $\boldsymbol{v}$-space, one obtains an effective equation for the isotropic component $f_{0}$ :

$$
\begin{array}{r}
\frac{\partial f_{0}}{\partial t}-\frac{\partial}{\partial \boldsymbol{x}} \cdot\left[\chi\left(\frac{\partial f_{0}}{\partial \boldsymbol{x}}-\boldsymbol{a} \frac{1}{v} \frac{\partial f_{0}}{\partial v}\right)\right]-\frac{1}{v^{2}} \frac{\partial}{\partial v}[\chi \\
{\left[\left(\boldsymbol{a} \cdot \boldsymbol{a} \frac{\partial f_{0}}{\partial v}-v \boldsymbol{a} \cdot \frac{\partial f_{0}}{\partial \boldsymbol{x}}\right)\right]} \\
=-C_{e e}\left[f_{0}, f_{0}\right]+S_{\mathrm{IB}} f_{0}
\end{array}
$$

using the notations $\boldsymbol{a}=e \boldsymbol{E} / m$, and $\chi(\boldsymbol{x}, v)=v^{2} / 6 \nu_{e i}(\boldsymbol{x}, v)$. Equation (5) is the so-called diffusive approximation ${ }^{11-13}$ to the Fokker-Planck equation. Let us point out, that in this last derivation contributions from $C_{e e}\left[f_{1}, f_{1}\right]$ have been neglected compared to $C_{e e}\left[f_{0}, f_{0}\right]$.

The relation for the self-collision operator is given by: ${ }^{14}$

$$
\begin{aligned}
& C_{e e}\left[f_{0}, f_{0}\right]=-\frac{Y_{e e}}{v^{2}} \frac{\partial}{\partial v}\left[I_{0} f_{0}+\frac{v}{3}\left(I_{2}+J_{-1}\right) \frac{\partial f_{0}}{\partial v}\right], \\
& \text { with } \quad I_{j}=\frac{4 \pi}{v^{j}} \int_{0}^{v} d v^{\prime} f_{0}\left(v^{\prime}\right) v^{\prime j+2}, \quad \text { and } J_{j}=\frac{4 \pi}{v^{j}} \int_{v}^{+\infty} d v^{\prime} f_{0}\left(v^{\prime}\right) v^{\prime j+2},
\end{aligned}
$$

with $Y_{e e}=4 \pi \ln \Lambda\left(e^{2} / 4 \pi \epsilon_{0} m\right)^{2}$. 
Note, that an external heating source $S_{\mathrm{IB}}$, representing inverse Bremsstrahlung between the laser light and the plasma, has been added to Eq.(5). The relation for $S_{\mathrm{IB}}$ has been derived in reference 4 :

$$
\begin{aligned}
S_{\mathrm{IB}} f_{0} & =\frac{A}{3}\left(\frac{v_{\mathrm{os}}}{v}\right)^{2} \frac{\partial}{\partial v}\left(\frac{g}{v} \frac{\partial f_{0}}{\partial v}\right), \\
g(v) & =\left[1+\left(\frac{v_{\omega}}{v}\right)^{6}\right]^{-1},
\end{aligned}
$$

where $A / v^{3}=\nu_{\mathrm{ei}}(v), v_{\mathrm{os}}=e E_{0} / \omega_{0}$ is the quiver velocity, $E_{0}$ the laser field amplitude, $\omega_{0}$ the laser frequency, and the velocity $v_{\omega}$ is defined such that $\nu_{\mathrm{ei}}\left(v_{\omega}\right)=\omega_{0} / 2$.

By taking the zeroth order velocity moment $\left(4 \pi \int_{0}^{+\infty} v^{2} d v\right)$ of equation (5), one obtains the continuity equation for the electron density:

$$
\begin{gathered}
\frac{\partial N_{e}}{\partial t}+\frac{\partial \boldsymbol{\Gamma}}{\partial \boldsymbol{x}}=0, \\
\text { with the particle flux: } \quad \boldsymbol{\Gamma}=-4 \pi \int_{0}^{+\infty} v^{2} d v \chi\left(\frac{\partial f_{0}}{\partial \boldsymbol{x}}-\boldsymbol{a} \frac{1}{v} \frac{\partial f_{0}}{\partial v}\right) .
\end{gathered}
$$

The condition of quasineutrality thus requires a divergence-free particle flux $\boldsymbol{\Gamma}$, which, in a one-dimensional system, can be reduced to:

$$
\boldsymbol{\Gamma} \equiv 0 \quad \Longleftrightarrow \quad \boldsymbol{a}=\frac{\int_{0}^{+\infty} v^{2} d v \chi \partial f_{0} / \partial \boldsymbol{x}}{\int_{0}^{+\infty} v^{2} d v(\chi / v) \partial f_{0} / \partial v}
$$

This last condition provides the equation for computing the self-consistent electrostatic field $\boldsymbol{E}$.

The heat equation is derived by taking the second order velocity moment $\left(4 \pi \int_{0}^{+\infty} v^{2} d v v^{2}\right)$ of $(5)$ :

$$
\frac{\partial}{\partial t}\left(\frac{3}{2} N_{e} T_{e}\right)+\frac{\partial \boldsymbol{q}}{\partial \boldsymbol{x}}=(-e) \boldsymbol{\Gamma} \cdot \boldsymbol{E}+\frac{4 \pi}{3} A m v_{\mathrm{os}}^{2} \int_{0}^{+\infty} d v \frac{\partial g}{\partial v} f_{0},
$$

with the heat flux: $\quad \boldsymbol{q}=-\frac{m}{2} 4 \pi \int_{0}^{+\infty} v^{2} d v v^{2} \chi\left(\frac{\partial f_{0}}{\partial \boldsymbol{x}}-\boldsymbol{a} \frac{1}{v} \frac{\partial f_{0}}{\partial v}\right)$.

\section{NUMERICAL IMPLEMENTATION}

Except for the ion dynamics, which are neglected here, equation (5) is identical to the Fokker-Planck equation considered by Epperlein in the code SPARK. ${ }^{13}$ The implementation 
of this equation was therefore achieved with essentially the same numerical methods as those presented in reference 13. For this reason, only a brief description of the numerics is given here, and we shall refer the reader to Epperlein's work for more details.

The Fokker-Planck equation (5) is solved here in one-dimensional configuration space, assuming either a slab or cylindrical geometry. In this way, phase space has been reduced to two dimensions: Position $x$ (slab) or $r$ (cylinder), and velocity amplitude $v$.

Reflective, fixed, and periodic boundary conditions are considered. Implementing open boundary conditions is a challenging problem in the case of the Fokker-Planck equation. Therefore, simulating an open system is effectively carried out by choosing the computation region large enough, so that no perturbation reaches the edge during the time of the simulation.

The alternating-direction-implicit (ADI) scheme enables one to efficiently solve the Fokker-Planck equation implicitly in time. For this approach to be applicable, Eq. must be written as a sum of differential terms operating separately on $\boldsymbol{x}$ and $v$ :

$$
\begin{aligned}
\frac{\partial f_{0}}{\partial t} & =\mathcal{L}_{\boldsymbol{x}} f_{0}+\mathcal{L}_{v} f_{0}, \\
\mathcal{L}_{\boldsymbol{x}} f_{0} & =\frac{\partial}{\partial \boldsymbol{x}} \cdot\left[\chi\left(\frac{\partial f_{0}}{\partial \boldsymbol{x}}+\boldsymbol{a} \alpha f_{0}\right)\right], \\
\mathcal{L}_{v} f_{0} & =\frac{1}{v^{2}} \frac{\partial}{\partial v}\left\{\chi\left(\boldsymbol{a} \cdot \boldsymbol{a} \frac{\partial f_{0}}{\partial v}+v \boldsymbol{a} \cdot \boldsymbol{\beta} f_{0}\right)+Y_{e e}\left[I_{0} f_{0}+\frac{v}{3}\left(I_{2}+J_{-1}\right) \frac{\partial f_{0}}{\partial v}\right]+\frac{A}{3} v_{\mathrm{os}}^{2} \frac{g}{v} \frac{\partial f_{0}}{\partial v}\right\} .
\end{aligned}
$$

The contribution to $\mathcal{L}_{v}$ proportional to $\chi$ is the so-called Ohmic term, which seems to be negligeable for most one-dimensional simulations. This is reflected by the fact, that its second order velocity moment leads to the Ohmic heating $(-e) \boldsymbol{\Gamma} \cdot \boldsymbol{E}$ in Eq. (6), which is zero as a result of the quasineutrality condition $\boldsymbol{\Gamma}=0$.

Notice that the cross-derivative terms with respect to $\boldsymbol{x}$ and $v$ appearing in Eq. (5) are now treated as non-linear terms, having defined $\alpha=-(1 / v) \partial \ln f_{0} / \partial v$, and $\boldsymbol{\beta}=-\partial \ln f_{0} / \partial \boldsymbol{x}$.

All non-linear terms in Eq. (7) have been dealt with iteratively in time. In other words, to advance the distribution by one time step $\Delta t$ from $f_{0}^{n}=f_{0}[t=n \Delta t]$ to $f_{0}^{n+1}=f_{0}[t=$ $(n+1) \Delta t]$, the coefficients of operators $\mathcal{L}_{\boldsymbol{x}, v}$ are first estimated with $f_{0}^{n}$. Having obtained a 
first estimate $\tilde{f}_{0}^{n+1}$ for $f_{0}^{n+1}$, using the ADI method, the step is repeated by estimating the coefficients of operators $\mathcal{L}_{\boldsymbol{x}, v}$ with $f_{0}^{n+}=\theta \tilde{f}_{0}^{n+1}+(1-\theta) f_{0}^{n}$. Here $\theta$ is the same implicitness parameter used in the ADI procedure (usually $\theta=1 / 2$ ).

Phase-space differentiation is carried out with a finite-difference scheme on a fixed Cartesian grid in $(\boldsymbol{x}, v)$. Various methods specifically developed for implementing the Landau self-collision operator ${ }^{15,16}$ have been applied. Non-equidistant meshes have been considered both in $\boldsymbol{x}$ and $v$. In cylindrical geometry for example, consecutive grid zones of a feathered radial mesh are such that $\Delta r_{i+1} / \Delta r_{i}=c$, with $c>1$. Such a mesh is particularly useful for simulating the heat transport transversely out of a single laser hot spot in an open system. Indeed, it provides high resolution within the laser beam, while the coarser mesh is sufficient far off axis, where phase-space presents little structure.

Typical numerical parameters used for the single hot spot simulations are $n_{r}=200$ spatial grid points in the radial direction, and $n_{v}=50$ points for the velocity mesh. For simulating open boundaries as described above, up to a thousand radial focal widths are considered for the total spatial size of the system. For velocity space, an interval of at least $6-8 v_{\text {th,max }}$ is chosen, where $v_{\text {th,max }}$ is evaluated at the maximum temperature estimated to be reached during the simulation. Time steps of the order $\Delta t \simeq 0.1 \nu_{e e}^{-1}$ provide very good temporal covergence.

\section{PARAMETER SCAN OF SINGLE LASER HOT SPOT SYSTEM}

The spatially one-dimensional Fokker-Planck code was applied for computing the electron heat transport transverse to the beam of a diffraction limited (i.e. single hot spot) laser, thus neglecting the transport parallel to the beam. This is justified in first approximation when considering the high intensity hot spot at the center of the focal region. Indeed, assuming a large focal length to beam diameter ratio $F$, this hot spot is very elongated along the beam: width $\sim F \lambda_{0}$, and length $\sim 8 F^{2} \lambda_{0}, \lambda_{0}$ being the wavelength of the laser light. ${ }^{17}$ 


\section{A. Parameter Space Covered by the Simulations}

As an initial conditions for the simulation, a uniform Maxwellian background plasma with temperature $T_{0}$ is assumed. This system is then driven, through inverse Bremsstrahlung, by a fixed laser intensity. The radial intensity profile $I(r)$ of the beam within the hot spot is approximated by a Gaussian profile:

$$
I(r)=I_{0} \exp \left[-\frac{1}{2}\left(\frac{r}{\Delta r_{\mathrm{FW}}}\right)^{2}\right]
$$

where $\Delta r_{\mathrm{FW}}$ is the radial focal width, of order $F \lambda_{0}$.

In the diffusive approximation, and assuming open boundary conditions, two independent parameters characterize the Fokker-Planck simulation of a single hot spot. This can easily be seen by writing equation (5) in normalized units, e.g. normalizing positions with respect to the thermal stopping length defined by $\bar{\lambda}_{\epsilon}=v_{\mathrm{th}} /\left[\nu_{\mathrm{ei}}\left(v_{\mathrm{th}}\right) \bar{\nu}_{\mathrm{ee}}\right]^{1 / 2}$, velocities with respect

to the background thermal velocity $v_{\mathrm{th}, 0}=\sqrt{T_{0} / m}$, and time with respect to the inverse of the background thermal self-collision frequency, defined here by $\bar{\nu}_{\mathrm{ee}}=(4 / Z) \nu_{\mathrm{ei}}\left(v_{\mathrm{th}}\right)=$ $6.91 \cdot 10^{-10} N_{\mathrm{cm}^{-3}} \ln \Lambda / T_{\mathrm{keV}}^{1.5}$. In the following, all quantities labeled with the index 0 are evaluated with the background temperature $T_{0}$ and peak intensity $I_{0}$.

The first of the two parameters measures the relative importance between velocity diffusion through self-collisions and spatial diffusion:

$$
\left[\frac{\text { Spatial Diffusion }}{\text { Self-Collisions }}\right]^{1 / 2} \sim \bar{\lambda}_{\epsilon, 0} / \Delta r_{\mathrm{FW}}=3.84 \cdot 10^{22} \frac{T_{0 \mathrm{keV}}^{2}}{\sqrt{Z} N_{\mathrm{cm}^{-3}} \ln \Lambda \Delta r_{\mathrm{FW} \mu \mathrm{m}}},
$$

with $\ln \Lambda$ standing for the Coulomb logarithm.

The second parameter measures the relative importance between self-collisions and inverse Bremsstrahlung:

$$
\frac{\text { Inverse Bremsstrahlung }}{\text { Self-Collisions }} \sim Z\left(v_{\mathrm{os}, 0} / v_{\mathrm{th}, 0}\right)^{2}=3.73 \cdot 10^{-16} \frac{Z I_{0 \mathrm{~W} / \mathrm{cm}^{2}} \lambda_{0 \mu \mathrm{m}}^{2}}{T_{0 \mathrm{keV}}} .
$$

Our goal was to include in the considered parameter range the conditions of the single laser hot spot experiments, ${ }^{5}$ carried out at the the Los Alamos National Laboratory (LANL). 
These conditions are given by: $\lambda_{0}=0.5 \mu \mathrm{m}$ laser light, focused by an $F / 7$ lens to a radial focal width $\Delta r_{\mathrm{FW}}=1.6 \mu \mathrm{m}$ [corresponding to a focal width half maximum diameter of $\left.d_{\mathrm{FWHM}}=(8 \ln 2)^{1 / 2} \Delta r_{\mathrm{FW}}=3.8 \mu \mathrm{m}\right]$, peak intensities in the range $I_{0}=2.5 \cdot 10^{14}-1.6 \cdot$ $10^{16} \mathrm{Wcm}^{-2}$, an electron density $N=2.23 \cdot 10^{20} \mathrm{~cm}^{-3}$ ( $=5 \%$ of critical density $N_{\mathrm{cr} \mathrm{cm}^{-3}}=$ $1.11 \cdot 10^{21} \lambda_{0 \mu \mathrm{m}}^{-2}$ ), and a background temperature $T_{0}=500 \mathrm{eV}$. Assuming a fully ionized $\mathrm{CH}$ (parylene-N) plasma, the effective degree of ionization is $Z=4.3$. Finally, choosing a Coulomb logarithm of the order of $\ln \Lambda=5$, these conditions lead to the following values for the relevant parameters: $\bar{\lambda}_{\epsilon, 0} / \Delta r_{\mathrm{FW}}=2.60$ and $Z\left(v_{\mathrm{os}, 0} / v_{\mathrm{th}, 0}\right)^{2}=0.201-12.83$.

On this basis, the actual parameters considered in the simulations were chosen as follows: To actually consider a certain range for spatial transport, the first parameter was varied between $\bar{\lambda}_{\epsilon, 0} / \Delta r_{\mathrm{FW}}=2.60 / 5^{4}-2.60 / 5^{0}$, by factors of 5 , while the second parameter was varied between $Z\left(v_{\mathrm{os}, 0} / v_{\mathrm{th}, 0}\right)^{2}=12.83 / 4^{3}-12.83 / 4^{0}$, by factors of 4 . In this scan, the conditions of the LANL experiment thus correspond to the cases with the largest spatial transport.

\section{B. Evolution of Temperature}

The single hot spot parameter scan was mainly performed in cylindrical geometry. All simulations were carried out up to the arbitrary time $t=500 \bar{\nu}_{\mathrm{ee}, 0}^{-1}$. For the LANL experimental conditions, this corresponds to a constant laser illumination in time for approximately 230ps, which is a somewhat longer drive than the 200ps Gaussian pulse considered in the experiment. It is however important to note, that the system at a given point remains essentially unchanged at later times over a very long period. Indeed, at least in the linear regime, it can be shown [from Eq.(24), Sec. V] that asymptotically in time the temperature at a fixed radius increases logarithmically, i.e. at an ever slower rate.

The evolution in time of the temperature variation $\Delta T$ at $r=0$ for the intensity $Z\left(v_{\mathrm{os}, 0} / v_{\mathrm{th}, 0}\right)^{2}=12.83 / 4^{0}$ and the different considered levels of spatial transport is shown in

Fig. 1. The final $\left(t=500 \bar{\nu}_{\mathrm{ee}, 0}^{-1}\right)$ temperature profiles are given in Fig. 2 for four different 
reference cases illustrating the limits of the considered parameter space: Cases (a) and (b) correspond both to the highest considered intensity $Z\left(v_{\mathrm{os}, 0} / v_{\mathrm{th}, 0}\right)^{2}=12.83 / 4^{0}$ and to the spatial transport levels $\bar{\lambda}_{\epsilon, 0} / \Delta r_{\mathrm{FW}}=2.60 / 5^{0}$ and $2.60 / 5^{4}$ respectively; same for cases (c) and $(\mathrm{d})$, but at the lowest considered intensity $Z\left(v_{\mathrm{os}, 0} / v_{\mathrm{th}, 0}\right)^{2}=12.83 / 4^{3}$. In the following, cases (a)-(d) will always refer to these four conditions. The final temperatures at $r=0$ for the whole parameter scan are plotted in Fig. 3.

For the highest spatial transport considered, i.e. $\bar{\lambda}_{\epsilon, 0} / \Delta r_{\mathrm{FW}}=2.60$, the actual simulations showed a temperature increase on the axis of the laser beam $(r=0)$ of 19.5\% (Fig. 2.a) with respect to the background temperature $T_{0}$ for $Z\left(v_{\mathrm{os}, 0} / v_{\mathrm{th}, 0}\right)^{2}=12.83 / 4^{0}$, and of just $1.8 \%$ for $Z\left(v_{\mathrm{os}, 0} / v_{\mathrm{th}, 0}\right)^{2}=12.83 / 4^{3}$ (Fig. 2.c). For theses cases of rapid spatial diffusion, the relative temperature increase in the last $200 \bar{\nu}_{\mathrm{ee}, 0}^{-1}$ period of the run is less than $0.1 \%$ for all considered intensities, indicating that the system has reached a quasi-steady state within the laser beam.

In the regime of low spatial transport, there is a larger temperature increase on axis over the simulation period (compare Fig. 2.b and 2.d with 2.a. and 2.c). For $\bar{\lambda}_{\epsilon, 0} / \Delta r_{\mathrm{FW}}=$ $2.60 / 5^{4}$ and highest intensity $Z\left(v_{\mathrm{os}, 0} / v_{\mathrm{th}, 0}\right)^{2}=12.83 / 4^{0}$, the temperature increase at $r=0$ at the end of the run is $\Delta T=6.3 T_{0}$. The relative increase in the last $200 \bar{\nu}_{\mathrm{ee}, 0}^{-1}$ interval is still $\sim 7 \%$ in this case (see Fig.1), so that the system has clearly not yet reached a quasisteady state at the end of the run. If the simulation was carried on, such a state would also ultimately be reached.

In fact, for sufficiently driven systems, there is a significant reduction of the laser light absorption at later times due to the non-linearities. Indeed, inverse Bremsstrahlung is essentially proportional to $f_{0}(v=0)$ [see Eq. (6)], which decreases as $T^{-3 / 2}$ with increasing temperature, and, as will be pointed out below, is further reduced due to the non-Maxwellian shape acquired by the distribution. Also, spatial transport is enhanced with increasing temperature, as $\lambda_{\epsilon}$ scales as $T^{2}$. These different non-linear effects thus further contribute to the system ultimately evolving at a slower rate within the laser beam. 


\section{Characterizing the Velocity Distribution Functions (VDFs)}

The velocity distribution functions on the beam axis at $t=500 \bar{\nu}_{\text {ee }, 0}^{-1}$ for cases (a)-(d) (as defined in Sec. IV B) are given in Fig. 4 and Fig. 5 in linear and logarithmic scale respectively. Inspired by the previous applications of Dum-Langdon-Matte (DLM) -type functions (also called super-Gaussians) for representing the distributions resulting from non-

classical drive and transport, ${ }^{4,3,2}$ the validity of such a representation was tested for the present results. These DLM distributions are of the form:

$$
f_{\mathrm{DLM}}(v ; n)=A_{n} \frac{N}{v_{\mathrm{th}}^{3}} \exp \left[-B_{n}\left(\frac{v}{v_{\mathrm{th}}}\right)^{n}\right],
$$

where the coefficients $A_{n}$ and $B_{n}$, ensuring the definition of density $\left(N=4 \pi \int_{0}^{\infty} v^{2} d v f\right)$ and temperature $\left(3 N v_{\mathrm{th}}^{2}=4 \pi \int_{0}^{\infty} v^{4} d v f\right)$, are given in terms of the gamma function $\Gamma$ by:

$$
A_{n}=\frac{1}{4 \pi} \frac{n}{\Gamma(3 / n)}\left[\frac{1}{3} \frac{\Gamma(5 / n)}{\Gamma(3 / n)}\right]^{3 / 2}, \quad \text { and } \quad B_{n}=\left[\frac{1}{3} \frac{\Gamma(5 / n)}{\Gamma(3 / n)}\right]^{n / 2} .
$$

Fitting such a DLM function $f_{\text {DLM }}$ to the velocity distribution $f_{0}$, obtained from the FokkerPlanck simulation, is performed in the following way: First, the density $N$ and thermal velocity $v_{\text {th }}$ in Eq. (9) are matched to those of $f_{0}$. The parameter $n$ is then varied so as to minimize the error:

$$
\operatorname{Error}(n)=4 \pi \int_{0}^{+\infty} v^{2} d v\left[f_{\mathrm{DLM}}(v ; n)-f_{0}(v)\right]^{2}
$$

As shown in Fig.4, the DLM functions provide a good representation for the bulk of the velocity distributions in all regimes. However, as can be seen in Fig. 5 , the fit $f_{\text {DLM }}$ systematically deviates from $f_{0}$ in the tail of the distribution for velocities above $v=3 v_{\text {th }}$, $v_{\text {th }}$ being the local thermal velocity. This disagreement between $f_{\text {DLM }}$ and $f_{0}$ is particularly significant in the high intensity cases (a) and (b), involving large values of the DLM parameter: $n=3.27$ and $n=3.85$ respectively (values given in Fig. 4). This deficiency of the DLM functions in representing the tail distribution of highly mobile particles is the result of spatial transport. 
In fact, spatial transport already affects the value of the DLM parameter $n$ itself. This is shown in Fig. 6, where $n$ is plotted as a function of the actual value of $Z\left(v_{\mathrm{os}} / v_{\mathrm{th}}\right)^{2}$ (i.e. evaluated at the local value of $v_{\text {th }}$ not $\left.v_{\text {th }, 0}\right)$ for the final state at $r=0$ of all simulations considered in the parameter scan. These results are compared to the relation established by Matte et al., valid for uniform illumination: ${ }^{3}$

$$
n_{\mathrm{Matte}}=2+\frac{3}{1+1.66 /\left[Z\left(v_{\mathrm{os}} / v_{\mathrm{th}}\right)^{2}\right]^{0.724}} .
$$

For fixed density and temperature, the fraction of particles at $v=0$ is reduced for increasing values of the parameter $n$. The ratio of $f_{\mathrm{DLM}}(v=0)$ compared to the value for an equivalent Maxwellian distribution is indicated for the different values of $n$ on the right hand side of Fig. 6. The absorption of the laser light through inverse Bremsstrahlung is thus reduced by the same factor.

In the presence of strong spatial transport $\left[\bar{\lambda}_{\epsilon, 0} / \Delta r_{\mathrm{FW}}=2.6\right.$, values labeled (A) in Fig. $6]$, and for all intensities, the parameter $n$ is significantly reduced from its value $n_{\text {Matte }}$ in the absence of spatial transport, essentially taking an intermediate value between $n=2$

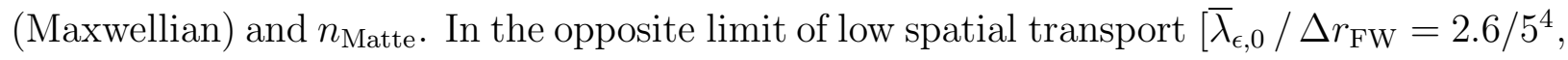
values labeled (E) in Fig. 6], the DLM parameter is expected to asymptotically tend towards Matte's curve. This is obviously the case in our simulations at low intensities. However, at high intensities, where the temperature $T$ on axis has significantly increased with respect to its background value $T_{0}$ (see Fig. 3 ), and the actual mean free path $\bar{\lambda}_{\epsilon}=\bar{\lambda}_{\epsilon, 0}\left(T / T_{0}\right)^{2}$ is therefore significantly amplified, this asymptotic limit would only be reached for levels of $\bar{\lambda}_{\epsilon, 0} / \Delta r_{\mathrm{FW}}$ below the ones considered in this parameter scan. Note that at intermediate levels of transport, the DLM parameter can take values above the ones predicted for uniform illumination.

Concerning the tails of the velocity distributions $f_{0}$, they are observed to be best represented for $v \gtrsim 3 v_{\text {th }}$ by a Maxwellian fit of the form:

$$
f_{\mathrm{M}, \text { tail }}\left(v ; N_{\text {tail }}, T_{\text {tail }}\right)=\frac{N_{\text {tail }}}{\left(2 \pi T_{\text {tail }} / m\right)^{3 / 2}} \exp \left[-\frac{1}{2} \frac{v^{2}}{T_{\text {tail }} / m}\right],
$$


where the density $N_{\text {tail }}$ and temperature $T_{\text {tail }}$ are in general different from the bulk values $N$ and $T$ obtained by the corresponding averages of $f_{0}$ over all velocities. Such a dependence of the tail is illustrated by Fig. 5 for cases (a)-(d), and has been checked for all simulations in the parameter scan.

The values $N_{\text {tail }}$ and $T_{\text {tail }}$ of the Maxwellian fit (10) were determined by minimizing the error:

$$
\operatorname{Error}\left(N_{\text {tail }}, T_{\text {tail }}\right)=\int_{3 v_{\text {th }}}^{5 v_{\text {th }}} d v\left\{\log \left[f_{\mathrm{M}, \text { tail }}\left(v ; N_{\text {tail }}, T_{\text {tail }}\right)\right]-\left[\log f_{0}(v)\right]\right\}^{2}
$$

Note that the fit was limited to the velocity interval $3-5 v_{\text {th }}$. Also, so as to provide a good fit over the many orders of magnitude over which the tails of the distributions may vary, the deviation between $f_{0}$ and $f_{\mathrm{M} \text {,tail }}$ is measured in terms of the logarithm of these functions.

In the cases of strong spatial transport, and for all intensities, the Gaussian-like tail of the velocity distribution on the beam axis is essentially identical to the tail of the Maxwellian background plasma (see Fig. 5.a and 5.c). This reflects the rapid thermalization with the surrounding plasma for large values of $\bar{\lambda}_{\epsilon, 0} / \Delta r_{\mathrm{FW}}$. A slight increase in the density $N_{\text {tail }}$ with respect to the background value $N_{0}$ is nonetheless observed in these cases, which, as verified, agrees well with the adiabatic response to the electrostatic potential drop:

$$
N_{\text {tail }} \sim \exp \left(-e \phi / T_{0}\right)
$$

where $\phi$ is the potential related to the self consistent electric field $\boldsymbol{E}=-\partial \phi / \partial \boldsymbol{x}$.

For low spatial transport (as in Fig. 5.b and 5.d), the tail temperature $T_{\text {tail }}$ takes an intermediate value between the local bulk temperature $T$ and the background temperature $T_{0}$. These values are also plotted in Fig. 3 for the entire parameter scan.

In order to combine the DLM fit (9) for the bulk of the distribution and the Maxwellian (10) for the tail into a single continuous function, valid for representing the whole distribution, the following global fit is defined:

$$
f_{\text {fit }}(v)=c(v) f_{\mathrm{DLM}}(v)+[1-c(v)] f_{\mathrm{M}, \mathrm{tail}}(v),
$$


where $c(v)$, ensuring the continuous transition from $f_{\mathrm{DLM}}$ to $f_{\mathrm{M} \text {,tail }}$ around the critical velocity $v_{c}=3 v_{\mathrm{th}}$, is arbitrarily chosen as:

$$
c(v)=\frac{1}{2}\left[1-\tanh \left(\frac{v-v_{c}}{\Delta v}\right)\right] .
$$

A transition width $\Delta v=5 \cdot 10^{-2} v_{\text {th }}$ agrees well with the numerical results. Except for a possible branch cut at $v=0$, a fit of the form (11) is in fact analytic, which turns out to be useful for solving the dispersion relation for the electron plasma waves, as will be seen in Sec. IV D. The quality of the global fit of type (11) for representing all regimes of the parameter scan is illustrated by the results in Figs. 4 and 5.

The contribution $Q_{r}(r, v)$ from each position in the phase space $(r, v)$ to the radial heat flux $q_{r}(r)$ is plotted in Fig. 7 for the final states of cases (a)-(d). More exactly, $Q_{r}$ is defined by

$$
Q_{r}(r, v)=-4 \pi^{2} m v^{4} r \chi\left(\frac{\partial f_{0}}{\partial r}-a_{r} \frac{1}{v} \frac{\partial f_{0}}{\partial v}\right)
$$

and such that

$$
2 \pi r q_{r}(r)=\int_{0}^{+\infty} d v Q_{r}(r, v)
$$

as is straightforward to verify from Eq. (6). Figure 7 clearly illustrates the outward heat flux carried by the more energetic particles, and a return current towards the beam axis at lower velocities. It is interesting to note, that the heat flux essentially takes place below the velocity $3 v_{\mathrm{th}}$, which is also the transition point between the DLM-like bulk and the Maxwellian tail of the velocity distributions. In the cases of strong spatial transport (Figs. 7.a and 7.c), the heat flux is essentially constant, at least up to the radius $r=10 \Delta r_{\mathrm{FW}}$ shown, reflecting the fact that the system basically reached a stationary state in this region. However, in the presence of weak spatial transport (Figs. 7.b and 7.d), the heat front has barely reached $r=5 \Delta r_{\mathrm{FW}}$, and consequently the system is still evolving at these radii. 


\section{Solving the Dispersion Relation for Electron Plasma Waves (EPWs)}

To quantify the effect of the non-Maxwellian velocity distribution functions on Landau damping, the linear dispersion relation for EPWs was solved:

$$
\epsilon(\omega, \boldsymbol{k})=0
$$

where $(\omega, \boldsymbol{k})$ are the frequency and wave vector of the mode, and $\epsilon$ is the local dispersion function evaluated for the distribution function $f_{0}(r, \boldsymbol{v} ; t)$ at a given radial position $r$ and time $t$ :

$$
\epsilon(\omega, \boldsymbol{k})=1-\frac{\omega_{p}^{2}}{k^{2}} \frac{1}{N} \int d \boldsymbol{v} \frac{\boldsymbol{k} \cdot \partial f_{0} / \partial \boldsymbol{v}}{\boldsymbol{k} \cdot \boldsymbol{v}-\omega}
$$

$\omega_{p}^{2}=N e^{2} / m \epsilon_{0}$ being the plasma frequency squared.

Making use of the fact that the considered distributions are isotropic in velocity space, the integral over the solid velocity angle can be carried out, which leads to:

$$
\epsilon(\omega, k)=1+\frac{\omega_{p}^{2}}{k^{2}} \frac{2 \pi}{N} \int_{-\infty}^{+\infty} d v \frac{k v}{k v-\omega} f_{0}(v), \quad \operatorname{Imag}(\omega)>0
$$

having symmetrized $f_{0}(v)$ around $v=0$.

Relation (13) for $\epsilon$ is only valid for positive imaginary frequencies (growing waves), but can be analytically continued into the lower complex frequency half-plane (damped waves). This can be achieved in the usual way, by deforming the contour of the velocity integral into the lower complex velocity half-plane, a so-called Landau contour (LC), so as to avoid the pole lying at $v_{0}=\omega / k$. This procedure naturally assumes that $f_{0}$ is itself analytic, and can be continued from the real axis to a region containing $v_{0}$. In this way:

$$
\begin{aligned}
\epsilon(\omega, k) & =1+\frac{\omega_{p}^{2}}{k^{2}} \frac{2 \pi}{N} \int_{\mathrm{LC}} d v \frac{k v}{k v-\omega} f_{0}(v) \\
& =1+\frac{\omega_{p}^{2}}{k^{2}} \frac{2 \pi}{N}\left[2 \pi i v_{0} f_{0}\left(v_{0}\right)+\int_{-\infty}^{+\infty} d v \frac{k v}{k v-\omega} f_{0}(v)\right], \quad \operatorname{Imag}(\omega)<0,
\end{aligned}
$$

where the last integral is again along the real axis, so that the Landau contour integral effectively leads to picking up the residue from the pole. The main practical problem of 
evaluating (14) for solving equation (12) consists in computing $f_{0}\left(v_{0}\right)$, and thus in finding the analytic continuation of the distribution $f_{0}$, which is provided by the Fokker-Planck simulation only for a finite number of mesh points $\left\{v_{j}\right\}_{j=1, \ldots, n_{v}}$ along the real velocity axis. This issue was resolved by making use of the analytic fit (11).

The whole issue of evaluating $f_{0}(v)$ in the complex plane is removed in the case of weakly damped modes, i.e. in the asymptotic regime of large wavelengths $\left(k \lambda_{D}, \ll 1\right.$, with $\lambda_{D}=v_{\text {th }} / \omega_{p}$ the Debye length), in which case the resonant approximation may be applied for solving the dispersion relation (12). The real frequency for the electron plasma waves is then given by the Bohm-Gross relation:

$$
\omega_{\mathrm{R}}^{2}=\omega_{p}^{2}\left[1+3\left(k \lambda_{D}\right)^{2}\right]
$$

and the damping rate by the value of $f_{0}$ at the resonant phase velocity $v_{\phi}=\omega_{\mathrm{R}} / k$ :

$$
\gamma / \omega_{p}=-\frac{\pi^{2}}{\left(k \lambda_{D}\right)^{3}} \frac{v_{\mathrm{th}}^{3}}{N} f_{0}\left(v_{\phi}\right)
$$

The growth rate $\gamma$ of the EPW as a function of the wave number $k$ has been computed for the distributions of the limiting cases (a)-(d) (velocity distributions shown in Figs. 4 and 5), and plotted in Fig. 8. For large values of $k \lambda_{D}$, these results were obtained numerically by directly solving the dispersion relation (12) in the complex frequency plane, making use of Eq. (14) for $\epsilon$ and the fit (11) for estimating $f_{0}$. For smaller values of $k \lambda_{D}$, the resonant approximation (15)-(16) was applied. As a reference, the dispersion relation was also solved considering three additional distributions for each case (a)-(d): The equivalent Maxwellian, the DLM distribution $f_{\mathrm{DLM}}$ fitting the bulk, and the DLM distribution from Matte's estimate (neglecting spatial transport) for the local value of $Z\left(v_{\mathrm{os}} / v_{\mathrm{th}}\right)^{2}$, providing the values $n_{\text {Matte }}=4.31,3.42,2.47$, and 2.28 for the DLM parameter in cases (a), (b), (c) and (d) respectively (see Fig. 6). As expected from relations (15) and (16), for each case (a)-(d) the differences between the various growth rates $\gamma$ essentially reflect the differences between the corresponding distributions plotted in Fig. 5. In the case of low spatial transport (see e.g. Fig. 8.b), the damping can thus be orders of magnitude lower for the actual 
velocity distribution compared to the equivalent Maxwellian. In the presence of strong spatial transport (Figs. 8.a and 8.b), however, the differences are much less dramatic.

\section{COMPARISON WITH NON-LOCAL HYDRODYNAMICS}

The non-local hydrodynamic approach, ${ }^{6-8}$ providing closure relations to the fluid equations for all regimes of collisionality, is a useful benchmark for the Fokker-Planck code. In turn, the full Fokker-Planck simulations enable one to determine quantitatively the limits of these relations, which are valid only in the linear regime of small deviations with respect to a Maxwellian background distribution. For this purpose, the evolution of the temperature profile in the case of a single laser hot spot was derived in the framework of the non-local hydrodynamic model, and compared to results from the non-linear Fokker-Planck code.

Assuming a Maxwellian background distribution driven by a low intensity laser, the heat equation (6) can be written in linearized form:

$$
\frac{\partial}{\partial t}\left(\frac{3}{2} N \delta T\right)+\frac{\partial \boldsymbol{q}}{\partial \boldsymbol{x}}=\bar{\nu}_{\mathrm{ei}} \frac{N}{N_{c}} \frac{I}{c}
$$

where $I=(1 / 2) \epsilon_{0} E_{0}^{2} c$ stands for the laser intensity, $N_{c}=\omega_{0}^{2} m \epsilon_{0} / e^{2}$ stands for the critical density, and the thermal electron-ion collision frequency is defined by $\bar{\nu}_{\mathrm{ei}}=$ $(2 / 3) \sqrt{2 / \pi} \nu_{\mathrm{ei}}\left(v_{\mathrm{th}}\right)$. So as to lighten notations for this linearized derivation, the label 0 refering to background quantities is omitted.

The non-local hydrodynamic approach provides the closure relations for the current $\boldsymbol{j}$ and heat flux $\boldsymbol{q}$, which can be written in Fourier representation with respect to space $\operatorname{as}^{8}$

$$
\begin{aligned}
& \boldsymbol{j}(\boldsymbol{k})=\sigma \boldsymbol{E}^{\star}+\alpha i \boldsymbol{k} \delta T+\xi_{j} \frac{e}{T} i \boldsymbol{k} \frac{N}{N_{c}} v_{\mathrm{th}} \bar{\lambda}_{\mathrm{ei}} \frac{I}{c}, \\
& \boldsymbol{q}(\boldsymbol{k})=-\alpha T \boldsymbol{E}^{\star}-\chi i \boldsymbol{k} \delta T+\xi_{q} i \boldsymbol{k} \frac{N}{N_{c}} v_{\mathrm{th}} \bar{\lambda}_{\mathrm{ei}} \frac{I}{c},
\end{aligned}
$$

with $\bar{\lambda}_{\mathrm{ei}}=v_{\mathrm{th}} / \bar{\nu}_{\mathrm{ei}}$ the thermal electron-ion mean free path, $\boldsymbol{k}$ the wave vector of the perturbation, and the effective force field being defined as $\boldsymbol{E}^{\star}=\boldsymbol{E}+(i \boldsymbol{k} / e)(\delta N T / N+\delta T)$. Note the following transport coefficients: The electric conductivity $\sigma$, heat conductivity $\chi$, 
thermoelectric coefficient $\alpha$, and the coefficients $\xi_{j, q}$ describing the coupling of the laser to the plasma. In general, these coefficients are not only function of the wave number $k=|\boldsymbol{k}|$, but also of the rate $\nu$ at which transport evolves. It has been shown, that this frequency dependence must be taken into account when considering the rapid relaxation of an initial temperature perturbation under conditions of strong non-local transport. ${ }^{18}$ However, for the driven systems studied here, the evolution takes place at a slower rate, and the transport coefficients at zero frequency $(\nu=0)$ computed in references 6-8 may therefore be considered.

From Eq. (18), the condition of quasineutrality $\boldsymbol{j}=0$ provides the equation for the self-consistent electric field

$$
\boldsymbol{E}^{\star}=-\frac{i \boldsymbol{k}}{\sigma}\left(\alpha \delta T+\xi_{j} \frac{e}{T} \frac{N}{N_{c}} v_{\mathrm{th}} \bar{\lambda}_{\mathrm{ei}} \frac{I}{c}\right) .
$$

The heat flux is then given by

$$
\boldsymbol{q}=-\kappa i \boldsymbol{k} \delta T+\xi i \boldsymbol{k} \frac{N}{N_{c}} v_{\mathrm{th}} \bar{\lambda}_{\mathrm{ei}} \frac{I}{c}
$$

with the effective heat conductivity $\kappa$ and coefficient $\xi$ defined by

$$
\begin{aligned}
& \kappa=\chi-\frac{T \alpha^{2}}{\sigma}, \\
& \xi=\xi_{q}+\frac{e \alpha}{\sigma} \xi_{j} .
\end{aligned}
$$

The linearized heat equation (17) can now be written in Fourier representation with respect to $\boldsymbol{x}$ as

$$
\frac{\partial}{\partial t} \delta T(\boldsymbol{k} ; t)=-\nu_{\text {relax }} \delta T(\boldsymbol{k}, t)+\frac{\bar{\nu}_{\mathrm{ei}}}{3}\left(\frac{v_{\mathrm{os}}}{v_{\mathrm{th}}}\right)^{2}(\boldsymbol{k})\left[1+\xi\left(k \bar{\lambda}_{\mathrm{ei}}\right)^{2}\right] T
$$

having used the notation $\nu_{\text {relax }}=(2 / 3) \kappa k^{2} / N$ for the relaxation rate of temperature perturbations, and the relation $I /\left(c N_{c}\right)=(T / 2)\left(v_{\mathrm{os}} / v_{\mathrm{th}}\right)^{2}$ between the laser intensity and the quiver velocity. It is straightforward to integrate (20) with respect to time, giving

$$
\delta T(\boldsymbol{k} ; t)=\frac{T}{3} \frac{\bar{\nu}_{\mathrm{ei}}}{\nu_{\text {relax }}}\left(\frac{v_{\mathrm{os}}}{v_{\mathrm{th}}}\right)^{2}(\boldsymbol{k})\left[1+\xi\left(k \bar{\lambda}_{\mathrm{ei}}\right)^{2}\right]\left[1-\exp \left(-\nu_{\text {relax }} t\right)\right]
$$


having assumed no initial temperature perturbation, and the laser drive invariant over time.

As in the numerical simulations, the heat transport is to be considered in the plane transverse to the laser beam. Considering a radially Gaussian laser intensity profile of the form (8), the two-dimensional Fourier transform becomes

$$
\begin{aligned}
\left(\frac{v_{\mathrm{os}}}{v_{\mathrm{th}}}\right)^{2}(k) & =\left(\frac{v_{\mathrm{os}, 0}}{v_{\mathrm{th}}}\right)^{2} \frac{1}{(2 \pi)^{2}} \int d \boldsymbol{x} \exp \left[-\frac{1}{2}\left(x / \Delta r_{\mathrm{FW}}\right)^{2}\right] \exp (-i \boldsymbol{k} \cdot \boldsymbol{x}) \\
& =\left(\frac{v_{\mathrm{os}, 0}}{v_{\mathrm{th}}}\right)^{2} \frac{\Delta r_{\mathrm{FW}}^{2}}{2 \pi} \exp \left[-\frac{1}{2}\left(k \Delta r_{\mathrm{FW}}\right)^{2}\right]
\end{aligned}
$$

According to Eqs. (21) and (22), one has $\delta T(\boldsymbol{k})=\delta T(|\boldsymbol{k}|)$, and, transforming back to direct space, the temperature perturbation becomes

$$
\begin{aligned}
\delta T(\boldsymbol{x} ; t) & =\int d \boldsymbol{k} \delta T(k ; t) \exp (i \boldsymbol{k} \cdot \boldsymbol{x})=\int_{0}^{+\infty} d k k \delta T(k ; t) \int_{-\pi}^{+\pi} d \theta \exp (i k r \cos \theta) \\
& =2 \pi \int_{0}^{+\infty} d k k J_{0}(k r) \delta T(k ; t)
\end{aligned}
$$

where $J_{0}$ is the zeroth order Bessel function.

Inserting (21) and (22) into (23), one finally obtains the radial temperature profile resulting from non-local heat transport:

$$
\begin{aligned}
\frac{\delta T(r ; t)}{T}=\frac{Z\left(v_{\mathrm{os}, 0} / v_{\mathrm{th}}\right)^{2} \Delta r_{\mathrm{FW}}^{2}}{9 \sqrt{2 \pi}} \int_{0}^{+\infty} d k k & J_{0}(k r) \exp \left[-\frac{1}{2}\left(k \Delta r_{\mathrm{FW}}\right)^{2}\right] \frac{\bar{\nu}_{\mathrm{ee}}}{\nu_{\mathrm{relax}}} \times \\
& {\left[1+\frac{9 \pi}{2} \frac{\xi}{Z}\left(k \bar{\lambda}_{\epsilon}\right)^{2}\right]\left[1-\exp \left(-\nu_{\text {relax }} t\right)\right] }
\end{aligned}
$$

having defined the thermal electron-electron collision frequency as $\bar{\nu}_{\mathrm{ee}}=(4 / Z) \nu_{\mathrm{ei}}\left(v_{\mathrm{th}}\right)$, and again using the thermal stopping length $\bar{\lambda}_{\epsilon}=v_{\mathrm{th}} /\left[\nu_{\mathrm{ei}}\left(v_{\mathrm{th}}\right) \bar{\nu}_{\mathrm{ee}}\right]^{1 / 2}=(1 / 3)(2 Z / \pi)^{1 / 2} \bar{\lambda}_{\mathrm{ei}}$.

For the transport coefficients $\kappa$ and $\xi$ appearing in (24), one can make use of the approximate relations

$$
\begin{aligned}
& \kappa(k, \nu=0)=\frac{\kappa_{0}}{1+\left(10 \sqrt{Z} k \bar{\lambda}_{\mathrm{ei}}\right)^{0.9}}=\frac{64}{\sqrt{2 \pi}} \frac{N \bar{\nu}_{\mathrm{ee}} \bar{\lambda}_{\epsilon}^{2}}{1+\left(15 \sqrt{2 \pi} k \bar{\lambda}_{\epsilon}\right)^{0.9}}, \\
& \frac{\xi(k ; \nu=0)}{Z}=\frac{34.5}{1+37\left[Z\left(k \bar{\lambda}_{\mathrm{ei}}\right)^{2}\right]^{0.67}}=\frac{34.5}{1+37\left[(9 \pi / 2)\left(k \bar{\lambda}_{\epsilon}\right)^{2}\right]^{0.67}}
\end{aligned}
$$

given by Eq.(3.27) in Ref. 7 and Eq.(33) in Ref. 8 respectively, and valid for high $Z$ and $k \bar{\lambda}_{\epsilon} \lesssim 1$ 
Note, that in general the interaction between the laser and the plasma not only leads to inverse Bremsstrahlung, but to ponderomotive effects as well; $\xi$ takes account of both of these dynamics. ${ }^{8}$ Also, one distinguishes between the isotropic and anisotropic (quadrupole) contribution of the laser polarization by decomposing this transport coefficient into $\xi=$ $\xi^{\text {iso }}+\left(3 \cos ^{2} \phi_{0}-1\right) \xi^{\text {aniso }}$, where $\cos \phi_{0}=\boldsymbol{E}_{0} \cdot \boldsymbol{k} /\left(E_{0} k\right)$. In equation (5), however, which is evolved in the non-linear Fokker-Planck simulations, ponderomotive and quadrupole effects of the laser light have been neglected. Relation (26) is consistent in this respect, as it corresponds only to the isotropic component of $\xi$, which has no ponderomotive contribution at zero frequency. Finally, the non-local transport coefficients computed in Refs 6-8 are valid for finite $Z$, while the approximate relations (25)-(26), function only of the stopping length $\bar{\lambda}_{\epsilon}$, correspond to the high $Z$ limit. This is again consistent with the diffusive approximation considered in the Fokker-Planck simulations.

The comparison between the non-local hydrodynamic approach and the Fokker-Planck simulations was carried out for a parameter scan similar to the one considered in Sec. IV. However, only the subset of transport levels $\bar{\lambda}_{\epsilon, 0} / \Delta r_{\mathrm{FW}}=2.60 / 5^{4}-2.60 / 5^{0}$, by factors of 25, was considered in this case. Also, to actually reach the linear regime with the FokkerPlanck code, lower laser intensities needed to be considered than in Sec. IV, so that the values $Z\left(v_{\mathrm{os}, 0} / v_{\mathrm{th}, 0}\right)^{2}=10^{-3}-10^{0}$, by factors of 10 , were chosen for the second relevant parameter. The simulations have again been run up to an arbitrary time, in this case $t=100 \bar{\nu}_{\mathrm{ee}, 0}^{-1}$. The final temperature profiles were then compared to relation (24). The corresponding results are summarized in Fig. 9.

Figure 9.a illustrates the final $\left(t=100 \bar{\nu}_{\mathrm{ee}, 0}^{-1}\right)$ temperature variation profiles from the simulations for the highest level of spatial transport $\bar{\lambda}_{\epsilon, 0} / \Delta r_{\mathrm{FW}}=2.60$ and for the different intensities considered. These profiles have been normalized with respect to the amplitude of the drive, i.e. $Z\left(v_{\mathrm{os}, 0} / v_{\mathrm{th}, 0}\right)^{2}$. Within the linear regime, these profiles should therefore coincide with the non-local hydrodynamic result obtained from Eq. (24), and which is also plotted in Fig. 9.a. This is clearly the case for intensities $Z\left(v_{\mathrm{os}, 0} / v_{\mathrm{th}, 0}\right)^{2}=10^{-3}$ and $10^{-2}$; significant deviations however appear at higher intensities. 
The differences in $\Delta T$, at $r=0$ and $t=100 \bar{\nu}_{\mathrm{ee}, 0}^{-1}$, between the linear non-local hydrodynamic result and the Fokker-Planck simulations, are given in Fig. 9.b as a function of $Z\left(v_{\mathrm{os}, 0} / v_{\mathrm{th}, 0}\right)^{2}$ and for the different levels of spatial transport. It turns out, that in the final state of the simulation, for all values of $\bar{\lambda}_{\epsilon, 0} / \Delta r_{\mathrm{FW}}$ considered, deviations of more than $10 \%$ have appeared for intensities above $Z\left(v_{\mathrm{os}, 0} / v_{\mathrm{th}, 0}\right)^{2}=10^{-2}-10^{-3}$.

The breakdown of the linearized fluid approach naturally occurs when the velocity distributions deviate too strongly from the initial Maxwellian background. This can be the result of one or both of the following: A too large (1) increase in temperature, (2) change of the actual shape of the distribution. In an attempt to quantify the limits of the non-local hydrodynamic approach in terms of these two effects, the temperature increase $\Delta T$ at $r=0$ in the final state of the simulation is plotted in Fig. 9.c, and the parameter $n$ of the DLM function, fitting the bulk of the distribution, plotted in Fig. 9.d. From Figs. 9.b-9.d, one can infer, that in the presence of temperature variations $\Delta T / T_{0} \gtrsim 1 \%$, and / or deformations from a Gaussian-shaped distribution characterized by DLM values $n \gtrsim 2.1$, the linearized hydrodynamic approach leads to errors larger than $10 \%$.

\section{MULTIPLE LASER HOT SPOT SYSTEMS}

In general, the non-linear simulation results obtained for single hot spots cannot be directly applied for describing systems composed of more than one such heat source. In a non-linear regime, as soon as the heat fronts from neighboring hot spots overlap, the principle of superposition of effects from the individual heat sources naturally breaks down.

As an illustration, let us consider such a multiple hot spot system. The cylindrical symmetry, used for carrying out the single hot spot calculations, becomes inappropriate for modeling two or more hot spots, and, so as to avoid the need for a fully two-dimensional spatial simulation, a slab-like geometry is assumed instead. Note that the change of geometry in itself affects the heat transport results. ${ }^{19}$

The system defined here is quite similar to the one considered by Afeyan et al.. ${ }^{2}$ However, 
the more realistic intensity profile considered in reference 2, corresponding to the speckle pattern produced by a random phase plate (RPP) laser beam, has been replaced by an equidistant series of equivalent hot spots. Each of these hot spots corresponds to what may be considered an average speckle in reference 2 . The actual simulation conditions are thus defined as follows: An initial Maxwellian background plasma is assumed at temperature $T_{0}=300 \mathrm{eV}$, and density $N=9.06 \cdot 10^{20} \mathrm{~cm}^{-3}$, corresponding to $10 \%$ critical for a laser light at $\lambda_{0}=0.35 \mu \mathrm{m}$. The ionization state is assumed to be $Z=20$ (fully stripped Ca). For these parameters, the background thermal stopping length is $\bar{\lambda}_{\epsilon, 0}=0.17 \mu m$ (taking again $\ln \Lambda \simeq 5$ ). The system simultaneously includes ten hot spots, their centers separated from each other by a distance of $20 \mu \mathrm{m}$, each having a Gaussian spatial dependence analogous to the one defined in cylindrical geometry by Eq. (8), with a radial focal width $\Delta r_{\mathrm{FW}}=3.3 \mu \mathrm{m}$. The peak laser intensity at each hot spot is $I_{0}=4.8 \cdot 10^{15} \mathrm{~W} \mathrm{~cm}^{-2}$, providing an average intensity over all ten speckles of $I_{\mathrm{av}}=2.0 \cdot 10^{15} \mathrm{Wcm}^{-2}$.

In a first simulation, the ten hot spots were placed at the center of a system $600 \mu \mathrm{m}$ wide, with fixed boundaries defined by the initial Maxwellian background. This system was then evolved for $t=4500 \bar{\nu}_{\mathrm{ee}, 0}^{-1}=235 \mathrm{psec}$, at which point it essentially reached an equilibrium with the fixed boundaries. The corresponding temperature profile is shown in Fig. 10.a. Thanks to the mirror symmetry of the laser intensity profile, only half of the system needed to be computed by taking a reflective boundary at the center.

Velocity distributions are plotted in Fig. 10.b and 10.c, in linear and logarithmic scale respectively. The distribution at the very center of the system $(x=0$, minimum in intensity profile) is compared to the one at $x=10 \mu \mathrm{m}$ (first maximum in intensity from center). As shown in Fig. 10.b, variations in the bulk of the distribution reflect the variations of the laser intensity profile. The DLM parameters providing the best fits at positions $x=0$ and $10 \mu m$ are $n=2.79$ and 3.10 respectively. The tails of the distributions are however essentially invariant within the heating region, as illustrated in Fig. 10.c. This is due to the fact that the slow particles of the bulk undergo heating locally, while the highly mobile particles in the tail are mainly submitted to dynamics resulting from an average intensity profile. This 
is confirmed by actually repeating the same simulation after having replaced the intensity profile from the ten hot spots by the average profile shown in Fig. 10.a. As appears in Fig. 10.c, the tail of the corresponding velocity distribution (taken arbitrarily at $x=0$ ) indeed matches the tails resulting from the spatially modulated intensity. Furthermore, as expected, the bulk distribution (Fig. 10.b), with a DLM fit value $n=2.85$, is intermediate between the distributions relative to minima and maxima in intensity of the multiple hot spot case.

Due to the large temperature increase by the end of the simulation (factor 10), the stopping length at the center of the system becomes of the order $\bar{\lambda}_{\epsilon} \simeq 17 \mu \mathrm{m}$ for thermal particles, and $\lambda_{\epsilon}\left(4 v_{\text {th }}\right)=4.4 \mathrm{~mm}$ for particles in the tail with velocities $v \simeq 4 v_{\text {th }}$. This is appreciably larger than the distance of $300 \mu \mathrm{m}$ from the center to the boundary of the initial simulation domain. One may therefore expect, that even at the very center of the system, the tails of the distribution are affected by the fixed boundary conditions. In an attempt to simulate open boundaries, two additional simulations were carried out by placing the edge of the computation domain at $2 \mathrm{~mm}$, and then $10 \mathrm{~mm}$ from the center of the system. Within the initial region of $300 \mu \mathrm{m}$ from the center, the results from these two last runs are essentially identical, thus proving that the intended goal of simulating open boundaries has been reached. These two runs are therefore not further distinguished. The corresponding temperature profile and distributions at time $t=4500 \bar{\nu}_{\mathrm{ee}, 0}^{-1}$ have also been plotted in Figs. 10.a and 10.c respectively. The tails of the distributions relative to the open boundary system are significantly less depleted than the ones for the fixed boundary case. The $10 \%$ variation in temperature at the center of the system, between the fixed and open boundary calculations, can only partly account for these differences in the distributions. Indeed, as can be seen in Fig. 10.c, the tails actually have different shapes: The distribution of the energetic particles is clearly Maxwellian $\left(T_{\text {tail }}=7.8 T_{0}\right)$ for open boundaries, while more super-Gaussian for fixed boundaries.

Finally, to provide a link between the multiple heat source simulations and the single hot spot calculations, a run was carried out by driving the system with the Gaussian intensity 
profile shown in Fig. 10.a. This single hot spot has a peak intensity $I_{0}=2.8 \cdot 10^{15} \mathrm{Wcm}^{-2}$, and a radial focal width $\Delta r_{\mathrm{FW}}=57 \mu \mathrm{m}$, which corresponds to the same total power and root mean square width as the square-shaped average intensity profile, and provides the following values for the relevant parameters: $\bar{\lambda}_{\epsilon, 0} / \Delta r_{\mathrm{FW}}=3.0 \cdot 10^{-3}$ and $Z\left(v_{\mathrm{os}, 0} / v_{\mathrm{th}, 0}\right)^{2}=8.3$. In this case, open boundaries were again considered. The corresponding temperature profile and velocity distribution at $x=0$ are also plotted in Fig. 10.a and 10.c respectively. These results are very similar to the ones from the multiple hot spot calculation with open boundaries. This example illustrates, how the single hot spot simulation results may provide average estimates for more complex systems.

\section{CONCLUSIONS}

Single laser hot spot simulations have been carried out over a wide dynamic range, characterized by the two relevant parameters $\bar{\lambda}_{\epsilon, 0} / \Delta r_{\mathrm{FW}}$ and $Z\left(v_{\mathrm{os}, 0} / v_{\mathrm{th}, 0}\right)^{2}$, which measure the level of spatial transport and inverse Bremsstrahlung respectively. These Fokker-Planck simulations have shown, that once the system has reached a quasi-steady state, the electron velocity distributions within the laser beam are well represented by a DLM-type function for the bulk, and a Maxwellian for the tail. Let us point out, that although most of the distributions shown in this paper were relative to the very center of the hot spot, they can be characterized in a similar way off axis.

Considering that DLM distributions provide good representations in the absence of transport, ${ }^{3}$ it is not astonishing that such functions remain good fits for representing the relatively slow moving particles of the bulk. Naturally, an equilibrium is established between the bulk distributions and the Maxwellian tails, which clearly are the result of spatial transport. The actual DLM parameter values fitting the bulk can therefore be significantly different from the estimates obtained using Matte's relation for uniform illumination. Both smaller (for strong spatial transport) and larger (for reduced transport) values have been observed. 
Under conditions of strong spatial transport, such as for the LANL single hot spot experiment, ${ }^{5}$ the Maxwellian tails within the laser beam are essentially identical to the distribution of the surrounding thermal plasma. For cases with weak spatial transport, the temperature of the Maxwellian tail at a given point is intermediate between the temperature of the corresponding bulk distribution and the temperature of the surrounding background. It is remarkable that the distributions present a Maxwellian tail over such a wide dynamical range. Although we have not yet been able to do so, an appropriately defined reduced model may enable to describe the underlying mechanism leading to this "universal" feature.

Although the single laser hot spot is a particular case of a non-uniformly driven plasma, we have shown that the corresponding results may also be useful for providing estimates of more complex systems. This was illustrated in the situation of multiple hot spots: Good estimates of the average velocity distribution function can be obtained by replacing the multiple heat sources by an appropriately chosen single hot spot.

As pointed out by Afeyan et al. ${ }^{2}$ the electron distribution tails depleted by non-classical drive may lead to orders of magnitude reductions of the linear Landau damping of plasma waves, which in turn may enable significantly lower thresholds and faster growth rates for SRS. Our results have mainly confirmed the possible reduction of linear Landau damping, although strong spatial transport, as well as different boundary conditions (open boundaries, versus fixed) can moderate this effect.

Finally, the non-linear Fokker-Planck simulations have also been applied for estimating the limits of the linear regime, under which the non-local hydrodynamic closure relations ${ }^{6-8}$ to the heat equation are valid. It has been shown, that perturbations of the initial Maxwellian background, corresponding to temperature variations as low as $\Delta T / T_{0} \gtrsim 1 \%$, and deformations of the Gaussian-shaped distribution characterized by DLM values $n \gtrsim 2.1$, lead to more than $10 \%$ inaccuracy of the hydrodynamic approach. 


\section{ACKNOWLEDGMENTS}

We wish to thank Ed Williams, Jim Albritton, and Dick Berger for their very useful comments in writing this paper.

This work was supported by the Lawrence Livermore National Laboratory under DOE

Interoffice Work Order Number B344523, by the U.S. Department of Energy Contract No. DE-AC02-76-CHO-3073. 


\section{REFERENCES}

${ }^{1}$ V. Yu. Bychenkov, W. Rozmus, and V. T. Tikhonchuk, Phys. Plasmas 4, 1481 (1997).

${ }^{2}$ B. B. Afeyan, A. E. Chou, J. P. Matte, R. P. J. Town, and W. J. Kruer, Phys. Rev. Lett. 80, 2322 (1998).

${ }^{3}$ J. P. Matte, M. Lamoureux, C. Möller, R. Y. Yin, J. Delettrez, J. Virmont, and T. W. Johnston, Plasma Phys. Controlled Fusion 30, 1665 (1988).

${ }^{4}$ A. B. Langdon, Phys. Rev. Lett. 44, 575 (1980).

${ }^{5}$ D. S. Montgomery, R. P. Johnson, H. A. Rose, J.A. Cobble, and J. C. Fernández, Phys. Rev. Lett. 84, 678 (2000).

${ }^{6}$ V. Yu. Bychenkov, W. Rozmus, V. T. Tikhonchuk, and A. V. Brantov, Phys. Rev. Lett. 75, 4405 (1995).

${ }^{7}$ A. V. Brantov, V. Yu. Bychenkov, V. T. Tikhonchuk, JETP 83, 716 (1996).

${ }^{8}$ A. V. Brantov, V. Yu. Bychenkov, V. T. Tikhonchuk, and W. Rozmus, Phys. Plasmas 5, 2742 (1998).

${ }^{9}$ J. F. Luciani, P. Mora, and J. Virmont, Phys. Rev. Lett. 51, 1664 (1983).

${ }^{10}$ J. R. Albritton, E. A. Williams, I. B. Bernstein, and K. P. Swartz, Phys. Rev. Lett. 57, 1887 (1986).

${ }^{11}$ I. B. Bernstein, Phys. Fluids 20, 577 (1976).

12 J. R. Albritton, Phys. Rev. Lett. 50, 2078 (1983).

${ }^{13}$ E. M. Epperlein, Laser and Particle Beams 12, 257 (1994).

${ }^{14}$ I. P. Shkarofsky, T. W. Johnston, and M. A. Bachynsky, The Particle Kinetics of Plasmas (Addison-Wesley, London, 1966).

15 J. S. Chang and G. Cooper, J. Comput. Phys. 6, 1 (1970). 
${ }^{16}$ A. B. Langdon, In CECAM Report of Workshop on The Flux Limiter and Heat Flow Instabilities in Laser-Fusion Plasmas (Université Paris Sud, France), p. 69.

${ }^{17}$ M. Born and E. Wolf, Principle of Optics (Pergamon Press, Oxford, 1975).

${ }^{18}$ S. Brunner, E. Valeo, and J. A. Krommes, Phys. Plasmas 7, 2810 (2000).

${ }^{19}$ V. Yu. Bychenkov, J. P. Matte, and T. W. Johnston, Phys. Plasmas 3, 3518 (1996). 
Fig. 1 Evolution in time of the temperature variation $\Delta T$ at $r=0$ for the peak intensity $Z\left(v_{\mathrm{os}, 0} / v_{\mathrm{th}, 0}\right)^{2}=12.83$, and for the different levels of spatial transport considered in the parameter scan.

Fig. 2 Temperature profiles (full line) at time $t=500 \bar{\nu}_{\mathrm{ee}, 0}^{-1}$, the final state of the simulation, for the four limiting cases of the parameter scan: Cases (a, b) and (c, d) correspond to the intensities $Z\left(v_{\mathrm{os}, 0} / v_{\mathrm{th}, 0}\right)^{2}=12.83 / 4^{0}$ and $12.83 / 4^{3}$ respectively. Cases (a, c) and (b, d) correspond to the transport levels $\bar{\lambda}_{\epsilon, 0} / \Delta r_{\mathrm{FW}}=2.6 / 5^{0}$ and $2.6 / 5^{4}$ respectively. The Gaussian intensity profile is plotted as a reference (dashed line).

Fig. 3 Increase $\Delta T$ of the bulk temperature (full line), at $r=0$ and in the final state $\left(t=500 \bar{\nu}_{\mathrm{ee}, 0}^{-1}\right)$ of the simulation, as a function of the different levels of transport $\bar{\lambda}_{\epsilon, 0} / \Delta r_{\mathrm{FW}}$, and for the different intensities $Z\left(v_{\mathrm{os}, 0} / v_{\mathrm{th}, 0}\right)^{2}$ considered in the parameter scan. The temperature increase $\Delta T_{\text {tail }}$ of the Maxwellian tail of the distributions is plotted with a dashed line [ $T_{\text {tail }}$ is defined in Eq. (10) Sec. IV C].

Fig. 4 Velocity distributions in linear scale at $r=0$ and $t=500 \bar{\nu}_{\mathrm{ee}, 0}^{-1}$ for the four limiting cases (a)-(d) of the parameter scan. The result from the Fokker-Planck (F-P) code is plotted with a full line, the fit of the form defined by Eq. (11) labeled with (o), the equivalent Maxwellian (same density and temperature as F-P result) with dashdotted line, and the background Maxwellian with dotted line. In this scale, the fit corresponds essentially to the contribution from the DLM-type function $f_{\mathrm{DLM}}$, with parameter value given by $n_{\mathrm{DLM}}$.

Fig. 5 Same as Fig. 4, but in logarithmic scale, and with an additional curve (dashed), corresponding to the DLM component $f_{\mathrm{DLM}}$ of the fit. These results clearly illustrate the transition around $v=3 v_{\text {th }}$ from the DLM-like bulk to the Maxwellian-like tails. The density $N_{\text {tail }}$ and temperature $T_{\text {tail }}$ of the Maxwellian fit $f_{\mathrm{M} \text {,tail }}$ are chosen such as to best represent the distribution in the velocity interval $3-5 v_{\text {th }}$. In cases (a) and (c) with strong spatial transport, the tail of the distribution is essentially identical to the 
tail of the Maxwellian background.

Fig. 6 Parameter $n$ of the DLM fit representing the bulk of the distribution at $r=0$ and $t=500 \bar{\nu}_{\mathrm{ee}, 0}^{-1}$ as a function of $Z\left(v_{o s} / v_{\mathrm{th}}\right)^{2}$, where $v_{\mathrm{th}}$ is evaluated at the same position and time. Labels A-E refer to the different levels $\bar{\lambda}_{\epsilon, 0} / \Delta r_{\mathrm{FW}}=2.6 / 5^{0}-2.6 / 5^{4}$ of spatial transport, and the successive curves correspond to the different levels of inverse Bremsstrahlung $Z\left(v_{\mathrm{os}, 0} / v_{\mathrm{th}, 0}\right)^{2}=12.8 / 4^{0}-12.83 / 4^{3}$ considered. As a reference, the relation for uniform illumination is plotted with a dashed line. The reduction of the number of particles at $v=0$ compared to a Maxwellian is indicated on the right hand side of the figure.

Fig. 7 Contribution $Q_{r}(r, v)$ to the heat flux from each point in the phase space $(r, v)$, for all four limiting cases (a)-(d). Contour levels plotted in full lines correspond to outward (positive) flux, while dashed lines correspond to return (negative) flux towards the beam axis; the dash-dotted line is the zero-flux contour level. Maximum and minimum value of $Q_{r}(r, v)$ are given in units $N_{0} T_{0} \bar{\nu}_{\mathrm{ee}, 0}^{-1} \Delta r_{\mathrm{FW}}^{2} / v_{\mathrm{th}}$. Also indicated is the velocity $3 v_{\text {th }}$, with $v_{\text {th }}$ evaluated at $r=0$.

Fig. 8 Damping rate $\gamma$ (in units of plasma frequency $\omega_{p}$ ) of the EPW as a function of the wave number $k$ (in units of inverse Debye length $\lambda_{D}$ ), for the four limiting cases (a)-(d). The curve labeled with (o) was obtained using the fit (11) to the Fokker-Planck code result. As a reference, the damping rates assuming the following distributions have also been plotted: Equivalent Maxwellian (dash-dotted line), DLM-type fit to the bulk (dashed line), and DLM distribution neglecting transport, i.e. estimating $n_{\mathrm{DLM}}$ with Matte's relation and the local value of $Z\left(v_{\mathrm{os}} / v_{\mathrm{th}}\right)^{2}$ (full line).

Fig. 9 Comparing non-linear Fokker-Planck (F-P) simulations with the linear non-local hydrodynamic approach, considering as a test case a parameter scan of the single laser hot spot system. All results plotted for time $t=100 \bar{\nu}_{\mathrm{ee}, 0}^{-1}$. Shown are (a) profiles of the temperature variation $\Delta T$ in the case $\bar{\lambda}_{\epsilon, 0} / \Delta r_{\mathrm{FW}}=2.60$ and for all intensities 
$Z\left(v_{\mathrm{os}, 0} / v_{\mathrm{th}, 0}\right)^{2}=10^{-3}-10^{0}$ considered, (b) relative difference in $\Delta T(r=0)$ between the F-P and the linear fluid results for the complete parameter scan, (c) absolute value of $\Delta T(r=0)$ for the F-P results, and (d) value of the DLM parameter fitting the bulk of the distributions.

Fig. 10 Results relative to multiple hot spot system. (a) Profiles of intensity, and final temperature. Only the right hand half of the system is shown. Three intensity patterns are considered: ten hot spots, an average square-shaped profile, and an equivalent single hot spot. Both fixed and open boundaries are considered. Velocity distributions for the different simulations are given in (b) linear and (c) logarithmic scale. 


\section{FIG.1 Brunner}

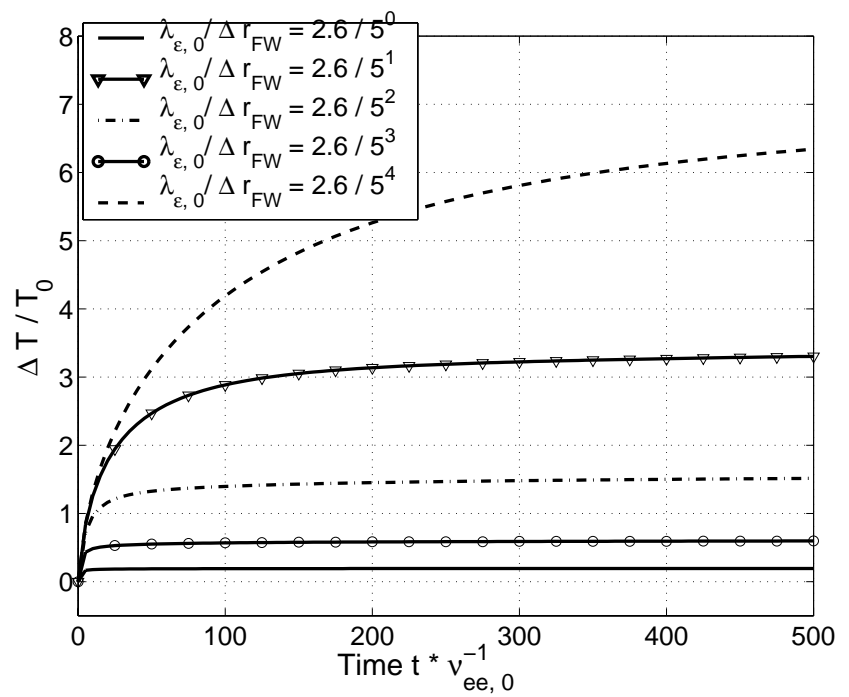




\section{FIG.2 Brunner}
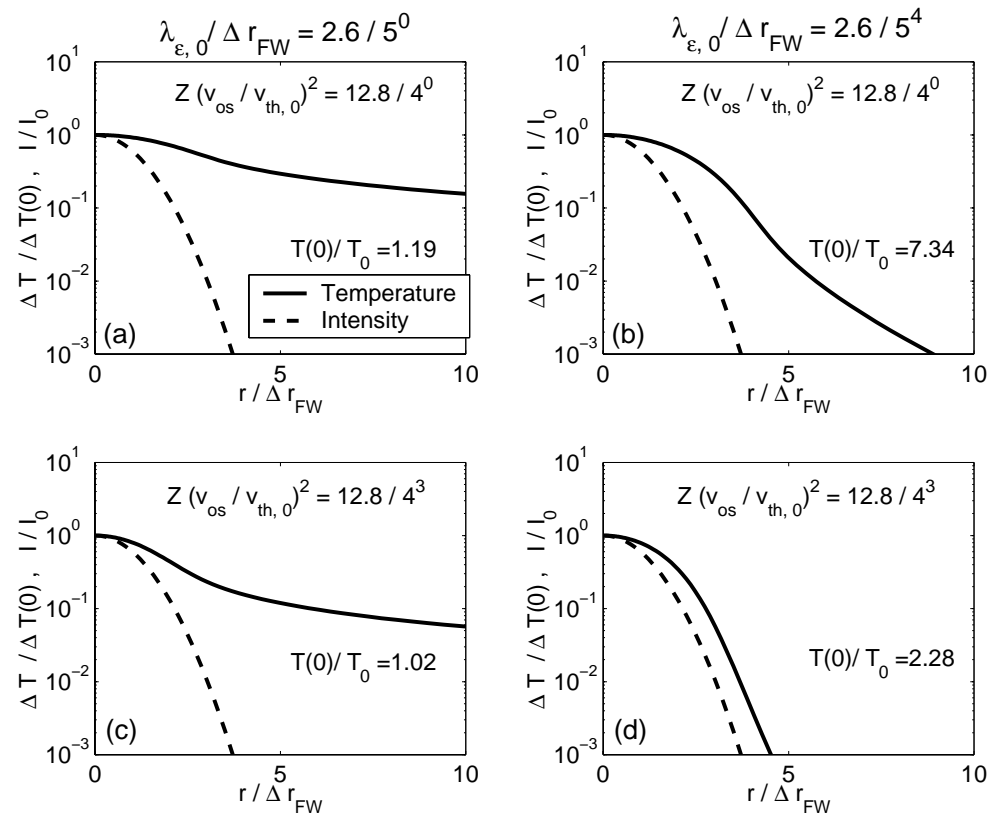


\section{FIG.3 Brunner}

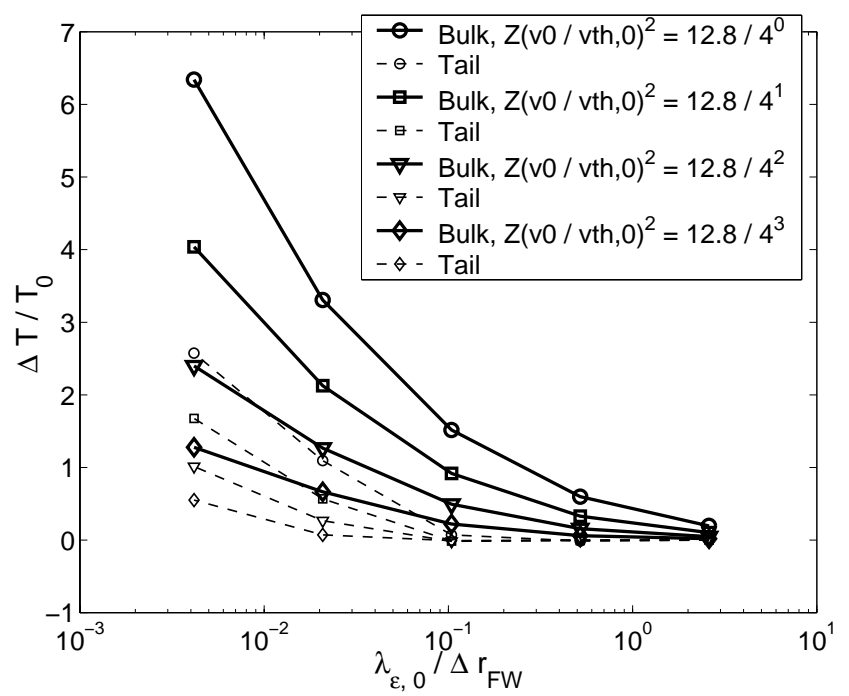




\section{FIG.4 Brunner}
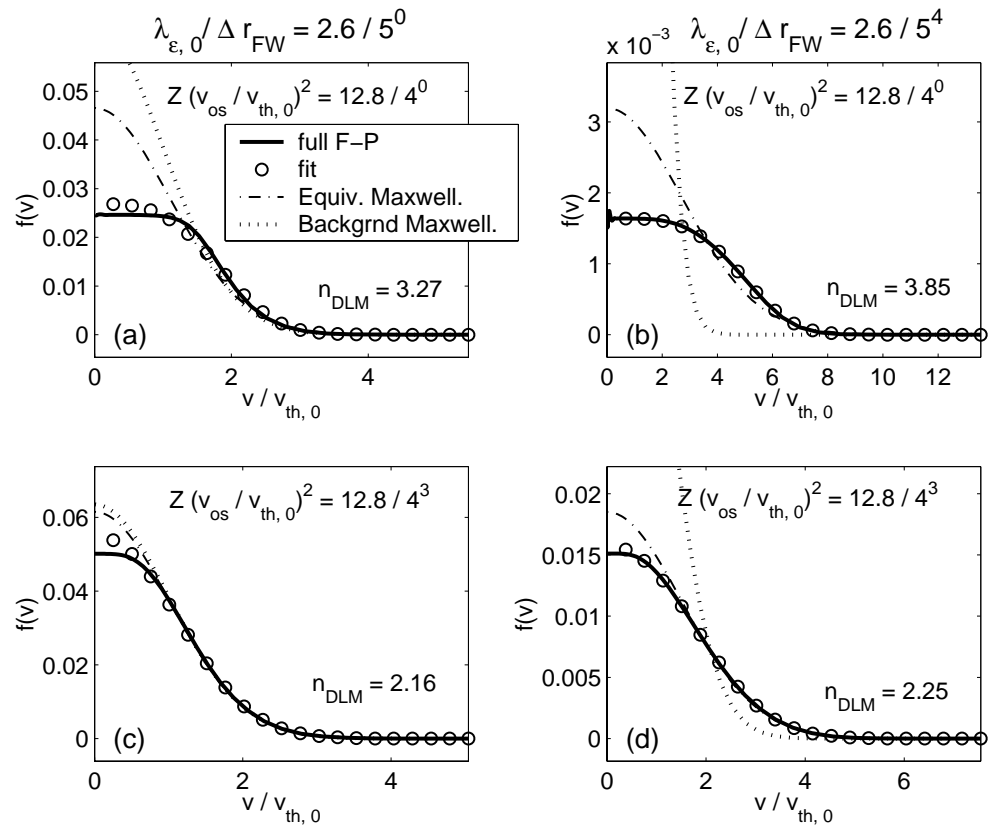


\section{FIG.5 Brunner}
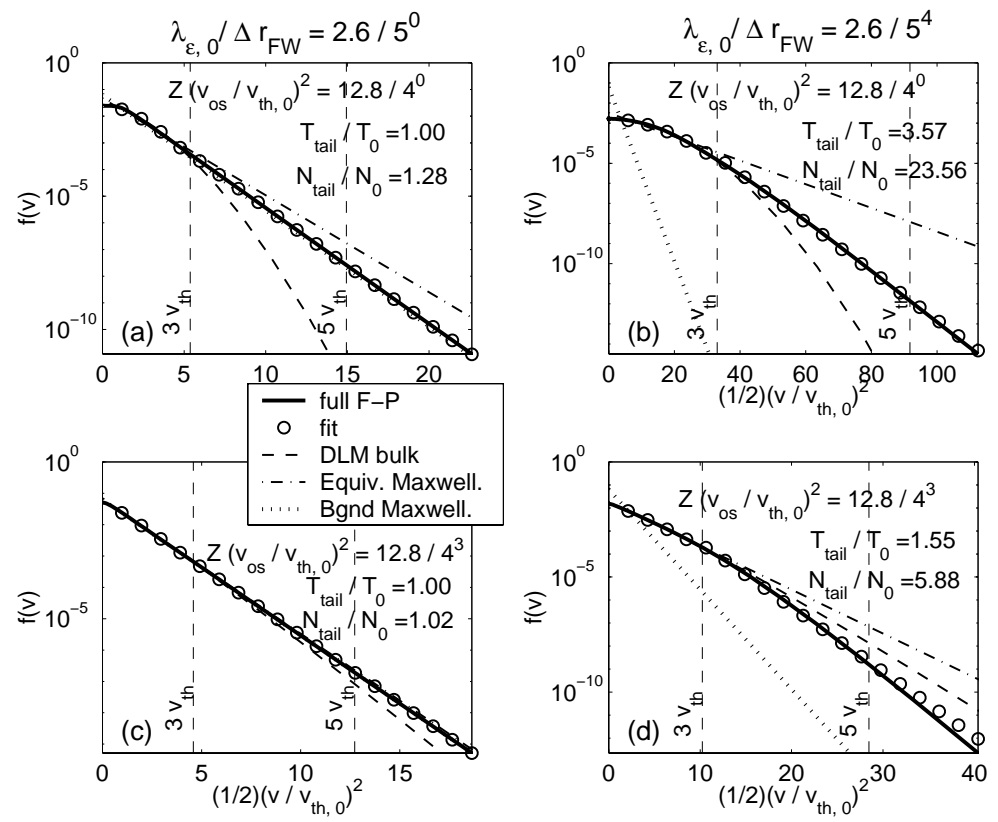


\section{FIG.6 Brunner}

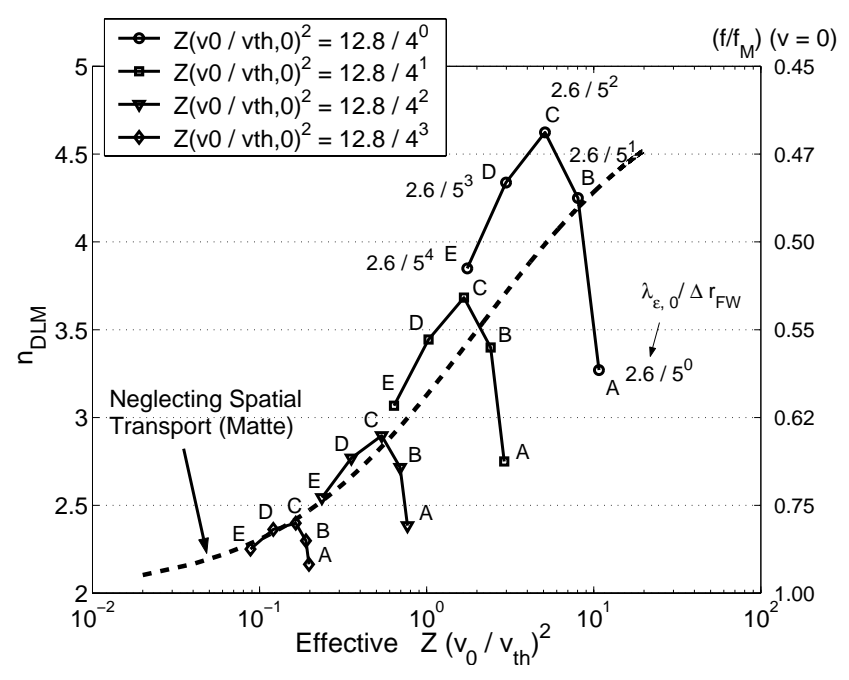




\section{FIG.7 Brunner}
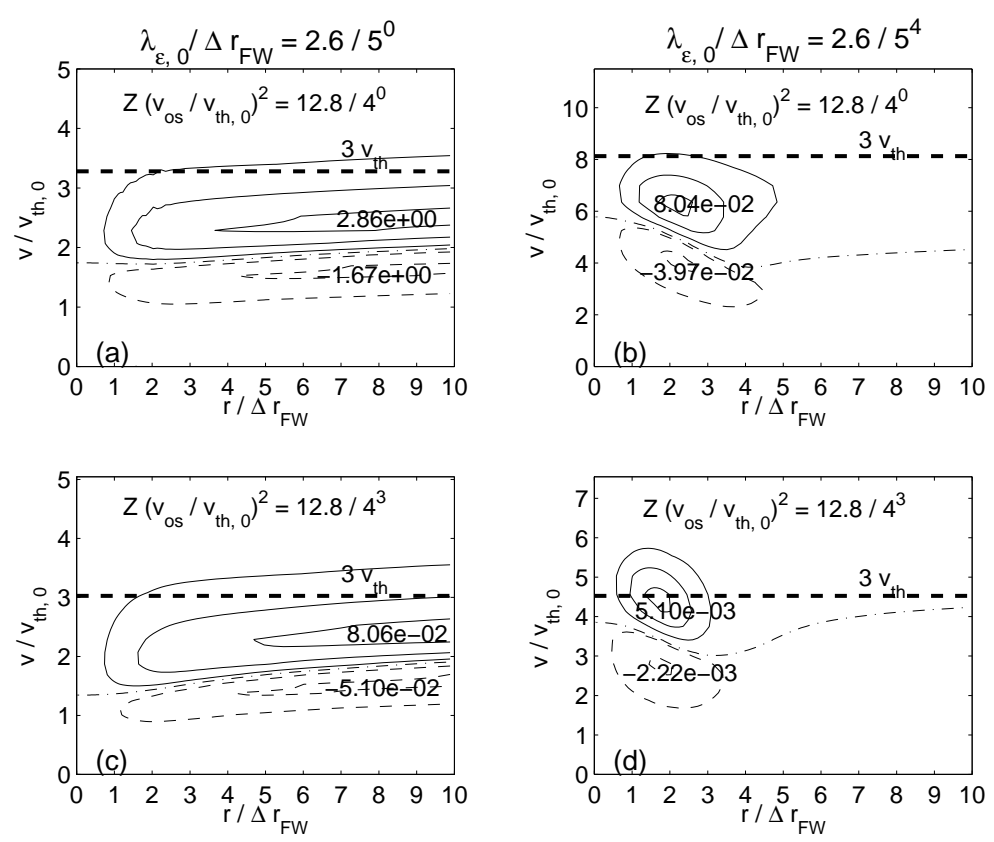


\section{FIG.8 Brunner}
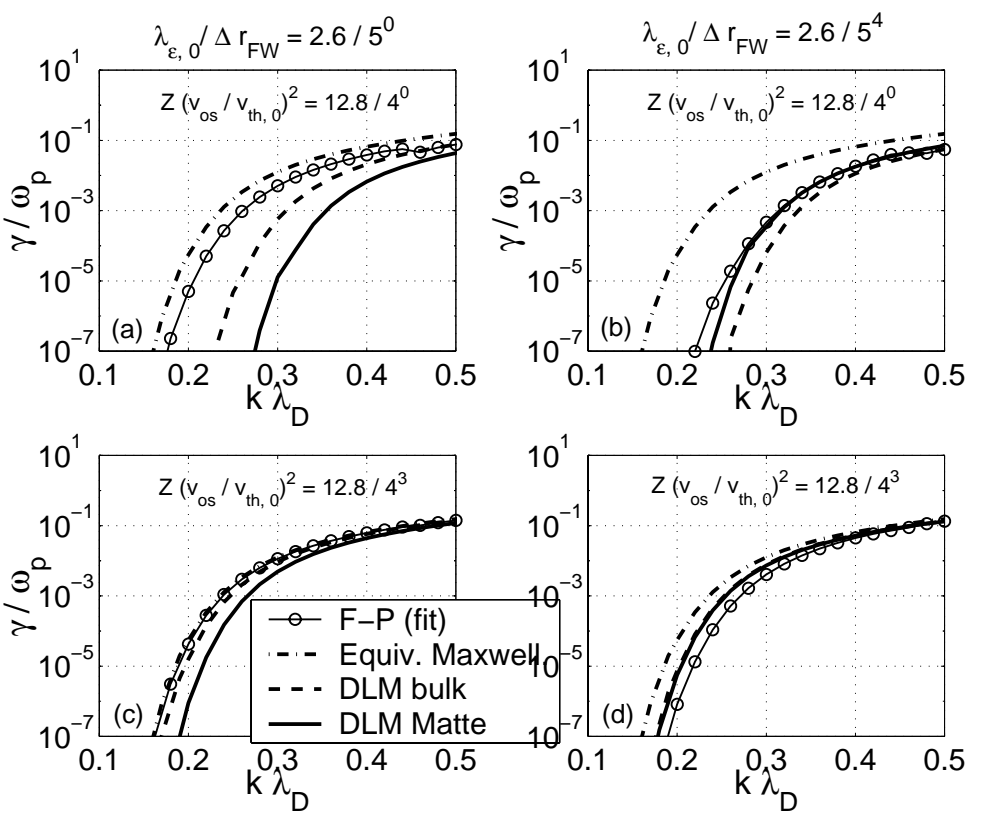


\section{FIG.9 Brunner}
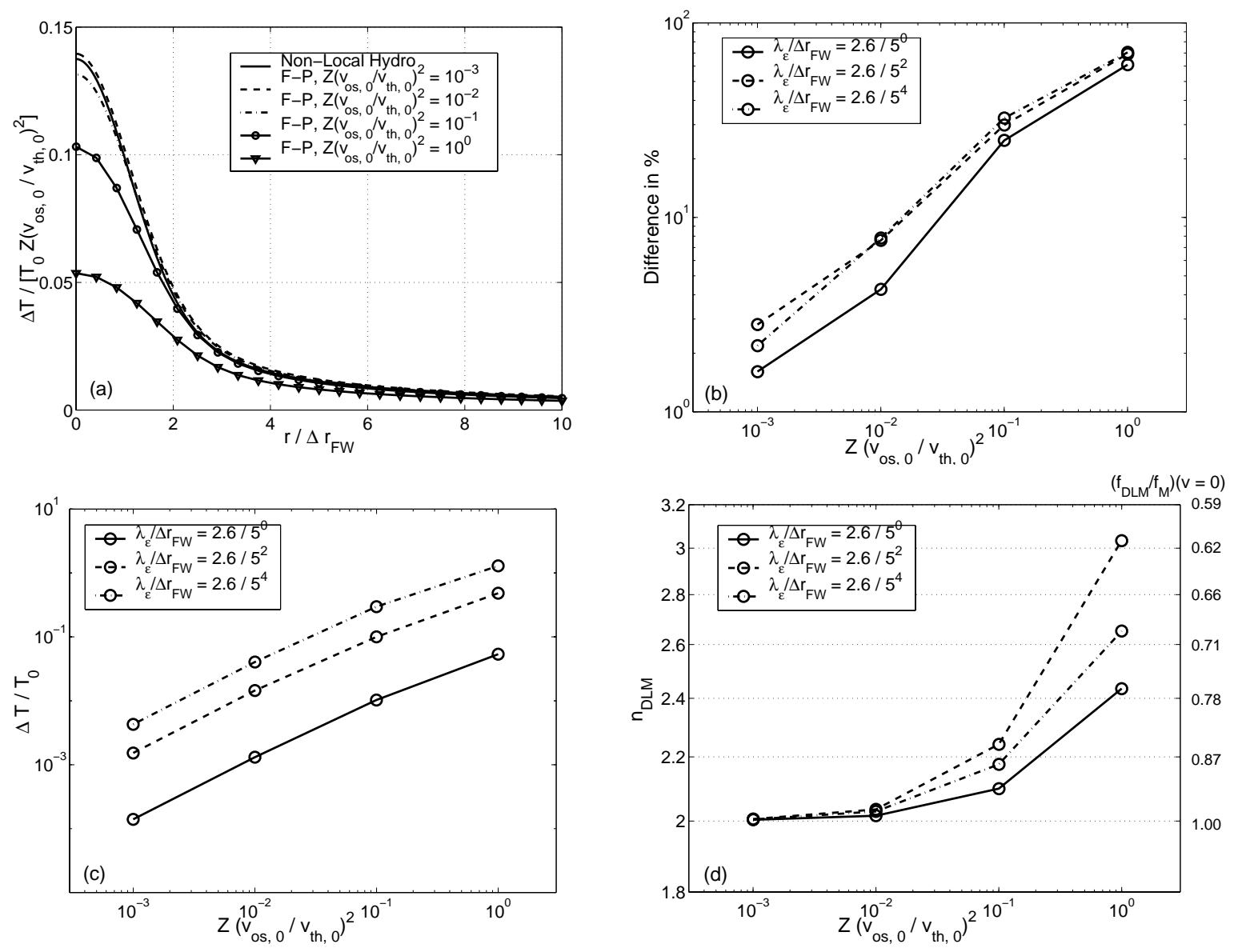


\section{FIG.10 Brunner}
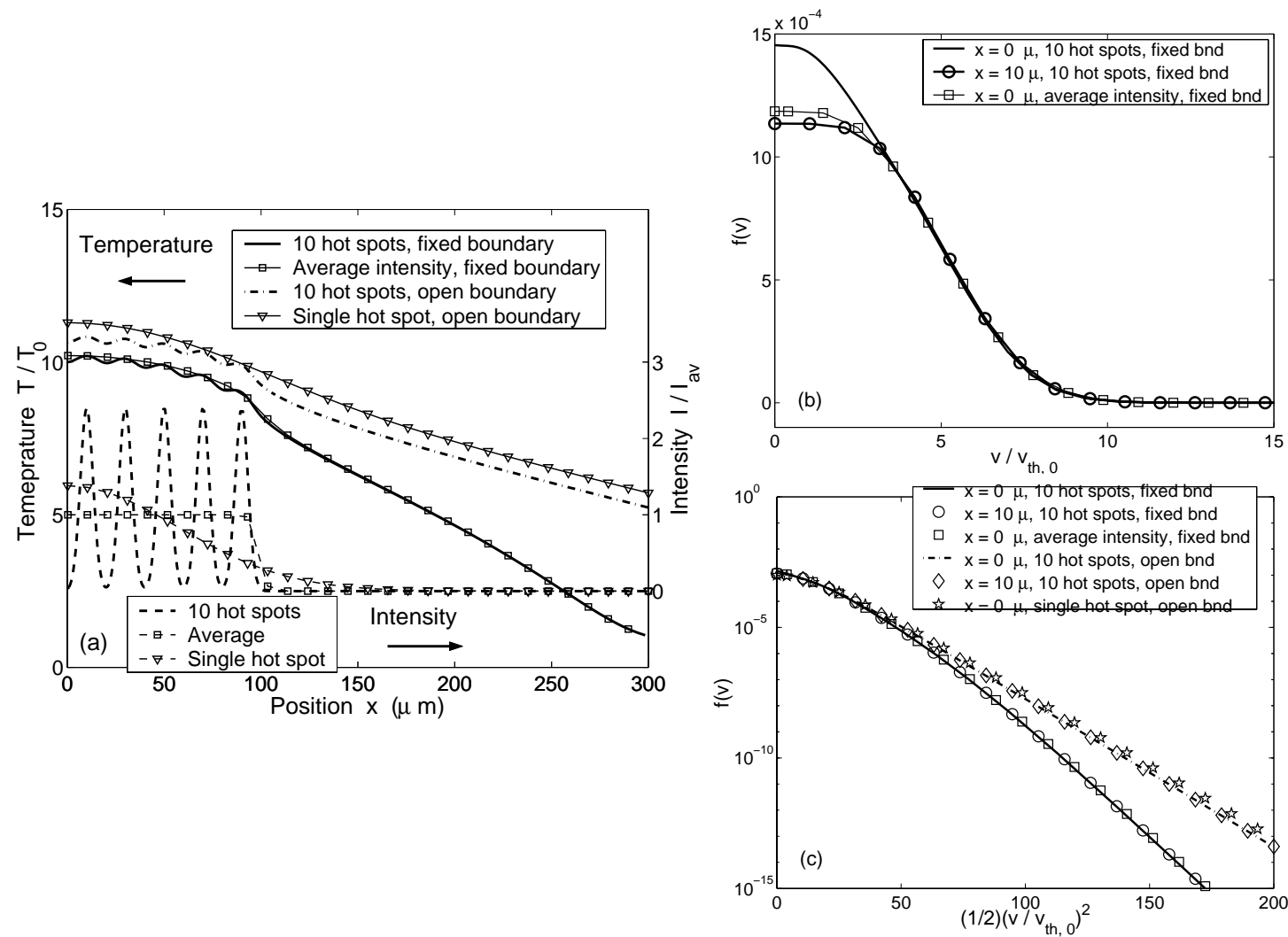


\section{PPPL Reports Disclaimer}

This report was prepared as an account of work sponsored by an agency of the United States Government. Neither the United States Government nor any agency thereof, nor any of their employees, makes any warranty, express or implied, or assumes any legal liability or responsibility for the accuracy, completeness, or usefulness of any information, apparatus, product, or process disclosed, or represents that its use would not infringe privately owned rights. Reference herein to any specific commercial product, process, or service by trade name, trademark, manufacturer, or otherwise, does not necessarily constitute or imply its endorsement, recommendation, or favoring by the United States Government or any agency thereof. The views and opinions of authors expressed herein do not necessarily state or reflect those of the United States Government or any agency thereof.

\section{Availability}

This report is posted on the U.S. Department of Energy's Princeton Plasma Physics Laboratory Publications and Reports web site in Calendar Year 2001. The home page for PPPL Reports and Publications is: http://www.pppl.gov/pub_report/

DOE and DOE Contractors can obtain copies of this report from:

U.S. Department of Energy

Office of Scientific and Technical Information

DOE Technical Information Services (DTIS)

P.O. Box 62

Oak Ridge, TN 37831

Telephone: (865) 576-8401

Fax: (865) 576-5728

Email: reports@adonis.osti.gov

This report is available to the general public from:

National Technical Information Service

U.S. Department of Commerce

5285 Port Royal Road

Springfield, VA 22161

Telephone: 1-800-553-6847 or

(703) 605-6000

Fax: (703) 321-8547

Internet: http://www.ntis.gov/ordering.htm 
The Princeton Plasma Physics Laboratory is operated by Princeton University under contract with the U.S. Department of Energy.

\author{
Information Services \\ Princeton Plasma Physics Laboratory \\ P.O. Box 451 \\ Princeton, NJ 08543
}

Phone: 609-243-2750

Fax: 609-243-2751

e-mail: pppl_info@pppl.gov

Internet Address: http://www.pppl.gov 